\title{
DYNAMICS OF TWO MUTUALLY \\ COUPLED SLOW INHIBITORY NEURONS
}

\author{
D. Terman ${ }^{1}, \quad$ N. Kopell ${ }^{2}$ and A. Bose ${ }^{3}$
}

1. Math Dept., Ohio State University, Columbus, Ohio 43210; terman@math.ohio-state.edu Supported in part by NSF grant DMS-9423796

2. Math. Dept. and Center for BioDynamics, Boston University, Boston, Ma. 02215; nk@math.bu.edu. $\quad$ Supported in part by NSF grant DMS-9200131 and NIMH grant MH47510

3. Dept.of Math., Center for Applied Mathematics and Statistics, New Jersey Institute of Technology, Newark, N.J. 07102; bose@nimbu.njit.edu

\begin{abstract}
Inhibition in oscillatory networks of neurons can have apparently paradoxical effects, sometimes creating dispersion of phases, sometimes fostering synchrony in the network. We analyze a pair of biophysically modeled neurons and show how the rates of onset and decay of inhibition interact with the time scales of the intrinsic oscillators to determine when stable synchrony is possible. We show that there are two different regimes in parameter space in which different combinations of the time constants and other parameters regulate whether the synchronous state is stable. We also discuss the construction and stability of non-synchronous solutions, and the implications of the analysis for larger networks. The analysis uses geometric techniques of singular perturbation theory that allow one to combine estimates from slow flows and fast jumps.
\end{abstract}

Key words: oscillations, inhibition, synchronization 


\section{Introduction.}

Synchronous activity in networks of inhibitory neurons has been observed in thalamic [22] and hippocampal [32] networks. Such activity has been the subject of a large number of simulation studies $[1,2,6,7,8,10,15,25,27,28,29,30,33]$. For coupled neural oscillators, a traditional view is that excitatory coupling leads to synchronous behavior, while inhibitory coupling leads to asynchronous behavior. Though this has been supported by many modeling studies $[4,11,18,19,20]$, there has recently been a variety of studies whose conclusion is the opposite [3, 4, 27, 28, 29]. Several of these papers emphasize the importance to the network behavior of the rates at which the synapses activate or deactivate. Some simple analytical models focus on the role of the rise time of the inhibition, and conclude that this rate must be sufficiently slow to obtain synchrony $[3,5,26]$. Others $[28,29]$, give simulations of coupled inhibitory neurons with a more biophysical basis, and obtain synchronous solutions when the inhibition decays at a rate slower than the rate at which the neurons recover in their refractory period.

The aim of this paper is to analyze a pair of biophysically modeled neurons, to understand how the rates of onset and decay of inhibition interact with the time scales of the intrinsic oscillators to determine when synchrony is possible. We show that there are two different regimes in parameter space in which different combinations of the time constants regulate whether the synchronous state is stable. Thus, a change of parameter that moves the system from one regime to the other changes which combination of parameters determines stability; this can be done by changing parameters such as synaptic reversal potential or maximal conductance, which do not directly change timing parameters.

The neurons that we analyze are relaxation oscillations, modeling either the envelope of activity of bursting neurons or neurons whose action potential is relatively broad (based on a calcium current rather than a sodium current). Without any coupling, each oscillator typically lies in either a silent or active phase, with rapid transitions between these that take place on a faster time scale. The synaptic coupling is modeled in a way similar to that in $[28,30]$. We focus on the cases in which the synapse activates and/or deactivates at a rate comparable to the rate at which the oscillator evolves in its silent or active phase. From the analysis, we can determine which combinations of time scales govern stability in different parameter regimes. Furthermore, we show why, and in what circumstances, the mutual interactions can lead to solutions that are more complicated than synchronous or antiphase [29]. These include suppressed solutions, in which one of the neurons oscillates while the other remains in its silent state, or solutions in which one oscillator may fire several times before the other does.

In other treatments of mutually inhibitory neurons [20,28], the concepts of "release" and "escape" have been useful in dissecting different mechanisms by which oscillations can occur in a network of two mutually inhibitory neurons. (See Discussion.) As shown in [20], the fast activation and deactivation of the synapse is critical to the above distinction. This paper shows that, even when the synapses have time scales comparable to the slow processes of the uncoupled neurons, the geometric techniques used to investigate the consequences of "escape" and "release" still work, though in higher dimensions and with potentially more complications. Thus, the analysis can be seen as placing the ideas of "release" and "escape" in a larger context, which includes slow synapses. 
The techniques of this paper are based on a geometric approach to singular perturbation problems. This approach allows us to dissect the entire flow into "fast" and "slow" pieces; these correspond to when the solution is in either its silent phase, active phase, or making a transition between these two phases. For each piece, we derive a reduced, lower order, system of equations. The reduced equations are analyzed to determine when we have expansion or compression over that piece. Combining these estimates then allows us to compute and analyze a "singular Poincare map", for which the limiting periodic orbit corresponds to a fixed point. For stability, we do not compute eigenvalues, but directly show that perturbations to the fixed point decay in time. All of our estimates are derived for when the singular perturbation parameter $\epsilon$ is formally set equal to zero; however, results found in [16], for example, show that our results also hold for $\epsilon$ sufficiently small.

In some previous studies (see $[14,24]$ ), the slow manifolds were one dimensional, and one could naturally define a metric between the two cells. The metric could either be a 'time-metric' which measures the time it takes for the 'trailing cell' to reach the position of the 'leading cell' or a 'space-metric' which measures the Euclidean distance between the two cells on the slow manifold. In the current paper the dimensions of the slow manifolds may be larger than one; this is the case when both cells are in their silent phase. There is no such natural metric when a slow manifold is higher dimensional. We will, in fact, need to define different metrics for different cases corresponding to different combinations of the parameters. Related techniques have been used by Terman and Lee [23] for slow synapses and by LoFaro and Kopell [14] for additional slow currents.

The outline of the paper is as follows: In Section 2, we give the equations for the full system, and the simplified equations that hold in each slow regime. We also give equations for a "direct" synapse, which is fast acting and an "indirect" synapse which has a delay to onset of inhibition. Those equations are used in Section 3, whose topic is the existence and stability of synchronous solutions. We show that the synchronous solution exists whenever the cells are oscillators, and can also exist if the cells are excitable (not oscillatory) provided that the inhibition decays slowly enough. We then address the question of what circumstances allow the synchronous solution to be asymptotically stable. We show that if the synapses between the cells are direct, then the synchronous solution is unstable - i.e. a delay in onset is necessary for the stability (also see [3, 5, 26]). For an indirect synapse, the answers are subtle. We show analytically that there are at least two combinations of parameters that affect the stability; these parameters include times that the cells are active and inactive, decay time of the synapse, and strength and reversal potential of the synapse. In different parameter regimes, only one or the other of the combinations is relevant to stability. We also show that the synchronizing effects of slowly decaying inhibition can phaselock heterogeneous cells not coupled to one another or to the cell providing the inhibition.

Even if the synchronous solution is stable, it may not be the only stable solution. The slow onset (or delay) in the inhibition provides a "window of opportunity" whose length partially determines the amount of difference in initial conditions that will decay toward synchrony. For a larger difference in initial conditions, the system displays different solutions. These include antiphase solutions, solutions in which one cell oscillates while the other is silent, and more exotic solutions in which one oscillates several times while the other is suppressed, and then the other fires. It is also possible that the network "crashes" 
and both become silent. In Section 4, we give simulations showing these other solutions and give heuristic explanations of how the parameters listed above interact to determine which solutions will be manifested.

The discussion in Section 5 includes a comparison of our results to those in related work. We also discuss potential implications of the analysis for larger networks. In particular, we give insights into the phenomenon of "clustering", in which subsets of cells synchronize and remain out of phase with other synchronized sets.

\section{The Model.}

\section{A. The single neuron.}

We model each individual neuron (or neural circuit), without any coupling, as the relaxation oscillator

$$
\begin{aligned}
v^{\prime} & =f(v, w) \\
w^{\prime} & =\epsilon g(v, w)
\end{aligned}
$$

Here $\epsilon$ is assumed to be small. We assume that the $v$-nullcline $f(v, w)=0$ defines a cubic-shaped curve as shown in Fig. 1, and the $w$-nullcline $g(v, w)=0$ is an increasing graph in the $v, w$ plane that intersects $f=0$ at a unique point $p_{0}$ (Fig. 1 ). We also assume that $f>0(f<0)$ below (above) the $v$-nullcline, and $g>0(g<0)$ below (above) the $w$-nullcline.

The analytical framework we develop applies for very general nonlinear functions $f$ and $g$. However, it will often be easier to interpret our results if we consider special forms for these nonlinear functions. The forms we choose are motivated by widely used models for neural systems. We sometimes assume that $f$ has the form

$$
f(v, w)=f_{1}(v)-g_{c} w\left(v-v_{R}\right)
$$

where $g_{c}>0$ and $v_{R}$ represent a maximal conductance and reversal potential, respectively. This includes the well-known Morris-Lecar equations [17]. (These equations are often used to describe the envelope of bursting neurons or the activity of a spiking neuron if the spikes have significant width.) In the Morris-Lecar equations, (2.2) gives the rate of change of cross-membrane potential as a sum of ionic currents and $w$ represents activation for a potassium current with $g_{c}=g_{K}$ and $v_{R}=v_{K}$.

We will sometimes assume that $g(v, w)$ has the form

$$
g(v, w)=\left(w_{\infty}(v)-w\right) / \tau(v)
$$

where $w_{\infty}(v)$ is nondecreasing in $v$ and $\tau(v)$ is positive. In (2.3), we are assuming that $w_{\infty}(v)=0$ on the left branch of the oscillator, and $w_{\infty}(v)=1$ on the right branch. We 
also assume that $\tau(v)$ is independent of $v$ on each of the two branches, so $\tau(v)=\tau^{-}$on the left branch and $\tau(v)=\tau^{+}$on the right one.

If the intersection $p_{0}$ of the nullclines lies on the left or the right branch of $f=0$, then $p_{0}$ corresponds to a stable fixed point of (2.1), providing that $\epsilon$ is small enough. If $p_{0}$ is on the left branch, we say that (2.1) is excitable. $p_{0}$ on the right hand branch corresponds to tonic firing for the unconnected neuron when the model is being used to describe the envelope of a neuron which is continually in the active phase. If $p_{0}$ lies on the middle branch of $f=0$, then (2.1) is oscillatory; there is a stable limit cycle, again if $\epsilon$ is sufficiently small. In the limit $\epsilon \rightarrow 0$, the periodic solution lies close to the singular periodic orbit shown in Fig. 1.

This singular orbit consists of four pieces, two slow and two rapid transitions between the slow pieces. We will refer to the parts of the trajectory on the left and right branches as the silent and active phases. These phases end when the trajectory reaches either the left knee or the right knee of $f=0$, initiating a rapid jump up to the active phase or jump down to the silent phase.

In the Morris-Lecar equations, $w$ represents the activation of a potassium current. In other models (including that considered in [28]), $w$ represents the inactivation of a calcium current, while $g_{c}$ and $v_{R}$ correspond to the maximal conductance and reversal potential of that current. The techniques developed in this paper apply to these other models. The precise stability conditions, however, depend on the particular form of the equations. This will be discussed further in Remark 11.

\section{B. Coupling.}

We model the pair of mutually inhibitory neurons by the following system of differential equations

$$
\begin{aligned}
v_{1}^{\prime} & =f\left(v_{1}, w_{1}\right)-s_{1} g_{s y n}\left(v_{1}-v_{s y n}\right) \\
w_{1}^{\prime} & =\epsilon g\left(v_{1}, w_{1}\right) \\
v_{2}^{\prime} & =f\left(v_{2}, w_{2}\right)-s_{2} g_{s y n}\left(v_{2}-v_{s y n}\right) \\
w_{2}^{\prime} & =\epsilon g\left(v_{2}, w_{2}\right)
\end{aligned}
$$

Here $\left(v_{1}, w_{1}\right)$ and $\left(v_{2}, w_{2}\right)$ correspond to the two oscillators, and $g_{s y n}$ is positive. The reversal potential $v_{s y n}$ is chosen so that if $(v, w)$ is on a singular solution, then $v>v_{\text {syn }}$; thus the synapse is hyperpolarizing (inhibitory). If $f(v, w)$ is given by $(2.2)$, then it is also natural to assume that $v_{R} \leq v_{\text {syn }}$.

The terms $s_{i}, i=1,2$, measure how the postsynaptic conductance depends on the presynaptic potentials $v_{j}, j \neq i$. For a "direct" synapse we assume that each $s_{i}$ satisfies a first order equation of the form

$$
s_{i}^{\prime}=\phi\left(1-s_{i}\right) H\left(v_{j}-\theta_{s y n}\right)-\epsilon K s_{i}
$$


where $j \neq i$. Here $\phi$ and $K$ are positive constants which do not depend on $\epsilon$, and $\theta_{\text {syn }}$ is the threshold above which one oscillator can influence another. Note that the synapse activates quickly (but not instantly), and deactivates slowly. This form of synaptic coupling has been considered in other neuronal models. See, for example, [28].

Synapses may be very complicated, involving, for example, secondary processes such as G-proteins. We shall refer to these as indirect synapses. Following [8], we model these by introducing new dependent variables $x_{1}$ and $x_{2}$, which satisfy first order equations. Each $x_{i}$ and $s_{i}$ satisfies the equations

$$
\begin{aligned}
x_{i}^{\prime} & =\epsilon \alpha\left(1-x_{i}\right) H\left(v_{j}-\theta_{v}\right)-\epsilon \beta x_{i} \\
s_{i}^{\prime} & =\phi\left(1-s_{i}\right) H\left(x_{i}-\theta_{s y n}\right)-\epsilon K s_{i}
\end{aligned}
$$

Here, $j \neq i$. The constants $\alpha$ and $\beta$ are assumed to be independent of $\epsilon$. The effect of the indirect synapses is to introduce a delay from the time one oscillator jumps up until the time the other oscillator feels the inhibition. For example, if the first oscillator jumps up, the secondary process is turned on when $v_{1}$ crosses the threshold $\theta_{v}$. The inhibition $s_{2}$ does not turn on until $x_{2}$ crosses $\theta_{\text {syn }}$; this takes a finite amount of (slow) time since $x_{2}$ evolves on the slow time scale, like $w_{i}$.

We will have to impose some technical assumptions on the parameters $\alpha$ and $\beta$. For example, if $v_{j}>\theta_{v}$, then $x_{i}$ will approach the fixed point $\alpha /(\alpha+\beta)$. We need to assume $\alpha /(\alpha+\beta)>\theta_{\text {syn }}$ so that $x_{i}$ can cross the threshold in order to turn on the inhibition. We assume this to be the case throughout the remainder of the paper. For our result concerning the stability of the synchronous solution, it will also be necessary to assume that $\alpha$ and $\beta$ are sufficiently large. Once again, this is necessary to guarantee that each cell's inhibition is able to turn on and turn off during each cycle.

\section{C. Fast and slow equations.}

For the coupled system, as for the uncoupled one, the slow regimes are those between the fast transitions, and in a slow regime each oscillator is either in a silent phase or an active one. To get the equations for any such slow regime, we rewrite the equations in terms of the slow time scale variable $\tau=\epsilon t$, and then set $\epsilon=0$ in the resulting equations. The reduced systems for the rapid transitions between silent and active phases are obtained (for the coupled or uncoupled system) simply by putting $\epsilon=0$ in (2.4) and (2.5) or (2.6). The notation $\dot{w}$ means $\frac{d w}{d \tau}$.

We now give the equations for the slow regimes.

i) Both oscillators in silent phase: If the synapse is direct, then the slow variables of this regime are $w_{i}$ and $s_{i}, i=1,2$. When both cells are in the silent phase, $v_{i}<\theta_{\text {syn }}$ and so the first term in the $\dot{s}_{i}$ equations is zero. Thus, in the limit $\epsilon=0$, the equations (2.4), (2.5) become: 


$$
\begin{aligned}
0 & =f\left(v_{i}, w_{i}\right)-s_{i} g_{s y n}\left(v_{i}-v_{s y n}\right) \\
\dot{w}_{i} & =g\left(v_{i}, w_{i}\right) \\
\dot{s}_{i} & =-K s_{i}
\end{aligned}
$$

where $i=1,2$. For an indirect synapse, each $x_{i}$ is also a slow variable, and we have the additional slow equation

$$
\dot{x}_{i}=-\beta x_{i}
$$

We can reduce the above system to equations for just the slow variables as follows. The first equation of (2.7) represents a cubic surface which we write as $v=h_{L}(w, s)$; in the silent phase, points lie on the left branch of this surface. Replacing the $v_{i}$ in the second equation of $(2.7)$ by $h_{L}\left(w_{i}, s_{i}\right)$ and setting $G_{L}(w, s) \equiv g\left(h_{L}(w, s), w\right)$, one obtains the following equations for the slow variables

$$
\begin{aligned}
\dot{w}_{i} & =G_{L}\left(w_{i}, s_{i}\right) \\
\dot{s}_{i} & =-K s_{i}
\end{aligned}
$$

For indirect synapses one has the additional equations (2.8). Here we are assuming that $x_{i}<\theta_{\text {syn }}$. Otherwise, we replace the second equation in (2.9) with $s_{i} \equiv 1$. In the simplified case of (2.3), (2.9) become

$$
\begin{aligned}
\dot{w}_{i} & =-w_{i} / \tau^{-} \\
\dot{s} & =-K s_{i}
\end{aligned}
$$

Note that in (2.7) or (2.9), the equations for the two cells are uncoupled; the coupling is through the positions when one or the other cell jumps. See [21].

The jump to a regime involving at least one of the cells excited happens when a trajectory of (2.9) hits a point at which one or both of the elements are at the up-jump curve. This curve, $w=w_{L}(s)$, is the $s$-dependent value of the point at which the "cubic"

$$
0=f(v, w)-g_{s y n} s\left(v-v_{s y n}\right)
$$

has a local minimum. We show later that $d w_{L}(s) / d s<0$. (See Remark 4 below.) This implies that the higher the value of the inhibition, the lower the value to which $w$ must decrease to jump. Fig. 2 shows sketches of the phase plane of (2.9) with the curve 
$w=w_{L}(s)$. In Fig. 2A, a single cell (as given by (2.1)) is oscillatory. There are then no fixed points of (2.9), and every trajectory arrives at the curve $w=w_{L}(s)$ in finite time. In Fig. 2B, a single cell is excitable with the fixed point $p_{0}$ lying on the left branch of the nullcline $f=0$. In this case, some of the solutions of (2.9) go to a stable critical point, and the system remains in the silent state. From (2.9), one can see that the rate of synaptic decay $K$, the rate of recovery $g(v, w)$ and the amount of inhibition all interact to determine the time and position of the jump to another process, or even if the system leaves the state in which both elements are silent. (See [23].) This will be discussed explicitly in Section 3.

ii) Both in active phase: First consider the direct synapse. When an element is in its active phase, it is producing the maximal amount of inhibition; the rise to $s=1$ happens on a fast time scale that is instantaneous for the slow equations. Thus, in the slow regime with both elements in active phase, both $s_{i}$ are set to 1 . There are then only two slow variables, $w_{i}$. Equations (2.7) become

$$
\begin{aligned}
0 & =f\left(v_{i}, w_{i}\right)-g_{\text {syn }}\left(v_{i}-v_{\text {syn }}\right) \\
\dot{w}_{i} & =g\left(v_{i}, w_{i}\right) \\
s_{i} & =1
\end{aligned}
$$

$i=1,2$. As before, we can express this system as equations for just the slow variables $w_{i}$. If we denote the right branch of the cubic surface defined by $(2.11)$ as $v=h_{R}(w, s)$ and let $G_{R}(w, s) \equiv g\left(h_{R}(w, s), w\right)$, then each $w_{i}$ satisfies

$$
\dot{w}=G_{R}(w, 1)
$$

In the simple case of $(2.3),(2.13)$ is

$$
\dot{w}=\left(1-w_{i}\right) / \tau^{+}
$$

For indirect synapses, the $x_{i}$ are also slow variables. Each of these satisfies the equation

$$
\dot{x}_{i}=\alpha\left(1-x_{i}\right) H\left(v_{j}-\theta_{v}\right)-\beta x_{i}=(\alpha+\beta)\left[\frac{\alpha}{\alpha+\beta}-x_{i}\right]
$$

Then (2.12) holds for $x_{i}>\theta_{\text {syn }}$. Otherwise, we replace the last equation in (2.12) by $s_{i}^{\prime}=-K s_{i}$.

A trajectory leaves this slow regime when one of the cells reaches the threshold for jumping down. Since $s_{i}$ is held at one, this threshold (the local maximum of the first equation of (2.12)) is a number $w_{R}(1)$, rather than a function of $s$. We assume that the nullcline $g(v, w)=0$ does not intersect any of the right branches of $(2.11)$ for $0 \leq s \leq 1$. 
This implies that a trajectory that jumps up to the active phase for both cells must eventually leave that slow regime.

For an indirect synapse, there is an extra slow regime corresponding to the rise of the $x$-variable. Assuming that each oscillator is active $\left(v_{i}>\theta_{v}\right)$, then as long as $x_{i}<$ $\theta_{\text {syn }},\left(v_{i}, w_{i}, s_{i}\right)$ satisfy (2.7). The difference between this and the equations of the silent phase is that $\left(v_{i}, w_{i}\right)$ now lies on the right branch of the cubic surface; hence, $w_{i}$ now satisfies

$$
\dot{w}_{i}=G_{R}\left(w_{i}, s_{i}\right)
$$

For $x_{i}>\theta_{\text {syn }}$, the equations for $v_{i}, w_{i}$ and $s_{i}$ switches to $(2.12)$.

iii) One cell active and one silent: This case arises when we consider antiphase solutions, for example. The derivation of the slow equations for each cell is very similar to the derivations already discussed. For this reason, we do not give the details.

\section{Main Results.}

\section{A. When is there a synchronous solution?}

We first construct the singular synchronous solution in the case of direct synapses. Such a solution must pass through the point at which both elements are on the edge of the upper plateau, whose $w$-component is $w_{R}(1)$. It is from this point that the cells jump down from the active to the silent phase. Provided that the trajectory can leave the process in which both are silent, the trajectory eventually jumps to the process in which both are active and returns to the starting point. The issue of existence of a synchronous solution thus reduces to the question of whether the jump to the active state is possible.

If the neurons are oscillatory, then the synchronous trajectory must reach the jumpup curve $w=w_{L}(s)$. This is true because $d w / d t<0$ if $s=0$ (uncoupled case) and because $s$ decreases. By continuity, $d w / d t<0$ for $s$ sufficiently small; since $s$ decreases, the trajectory is eventually in a region where $w$ must decrease until it hits $w_{L}(s)$.

If both cells are excitable, the existence of a synchronous solution depends on the rate $K$ of decay of the inhibition. If $K$ is large, the decay is fast; for $K$ large enough, the system behaves in this slow regime like the system with $s=0$, in which the critical point of the silent regime prevents the trajectory from leaving this regime. Hence, no periodic solution is possible.

The surprising case is $K$ sufficiently small, provided that the cells are oscillatory for some fixed values of $s$. We note that this is indeed possible for the Morris-Lecar type models given in the Appendix. One might think of such a system as a 2-D caricature of larger systems that have additional currents, such as the $I_{h}$ [12]. The following argument shows that the synchronous trajectory does leave the "both silent" regime, and hence there is a periodic solution. Fig. 3A shows the $(w, s)$ phase plane for $(2.9)$ for $K=0$. The curves intersecting $w=w_{L}(s)$ are the critical points of (2.9) at $K=0$, i.e., the equilibrium points for $w$ when $s$ is frozen. In this example, there are two such curves; these are labeled as 
$w_{c}^{+}$and $w_{c}^{-}$in Fig. 3. Note that there is an interval of values of $s$ between these curves for which the trajectory is not cut off from the threshold by a critical point, so oscillations can occur, even when $K=0$.

For $K>0$, trajectories start in the silent regime with $s=1$, and both $w$ and $s$ begin to decrease, as shown in Fig. 3B. If $K$ is sufficiently small, then $(w, s)$ must reach the upper curve $w_{c}^{+}$of critical points of the $K=0$ system. For $K>0$, this curve represents the $w$-nullcline of (2.9). Hence, $w$ begins to increase after $(w, s)$ crosses this curve. Since $s$ continues to decrease, and $(w, s)$ can never cross this curve again, it follows that $(w, s)$ must eventually leave the slow regime along a point on $w_{L}(s)$.

The construction of the synchronous solution for the case of indirect synapses is very similar to that for direct synapses except we must now be concerned with the additional slow variables $x_{1}$ and $x_{2}$. We may start this solution with each $\left(v_{i}, w_{i}, s_{i}\right)$ at the same jump-down point; however we must also choose the initial value $x_{1}(0)=x_{2}(0)$ so that after one complete cycle, each $x_{i}(t)$ returns to this value. As we shall see, this is equivalent to showing that a certain return map possesses a fixed point. The return map is formally defined in Section 3D; it is shown in Section 4 that the map is well defined and gives rise to a fixed point as long as the synchronous trajectory is able to leave the process in which both cells are silent. This is precisely the condition needed for the existence of the synchronous solution for the case of direct synapses. Hence, as before, the synchronous solution exists if the neurons are oscillatory, or if $K$ is small and the cells are oscillatory for some fixed values of $s$.

\section{B. Instability of the synchronous solution for direct synapses.}

We show in this section that the synchronous solution is not stable when the synapse is direct. In the next section, we state results which show that the synchronous solution may be stable if the synapses are indirect. The stability depends on whether certain relationships between the parameters in (2.4), (2.6) are satisfied.

Suppose that the synapse is direct. We start with both oscillators in the silent phase, and assume that cell 1 reaches the jump-up curve $w_{L}(s)$ first. When cell 1 jumps, the other begins to feel inhibition as $v_{1}$ crosses $\theta_{\text {syn }}$. For the direct synapse, this inhibitory conductance, $s_{2}$, jumps instantly (in the slow time scale) to $s_{2}=1$. The effect of this on the $v_{2}, w_{2}$ equations is to instantly move the second cell away from its threshold. This is shown in Fig. 4, where the two cells are denoted by $C_{1}$ and $C_{2}$. The amount that it is removed from the threshold stays bounded away from zero no matter how close the second cell was originally to the first one. Thus, infinitesimally small perturbations get magnified at this stage of the dynamics to finite size. Though the points can get somewhat closer as they transverse the rest of the cycle, they remain a finite distance apart over the cycle. This shows that small perturbations are expanded by the dynamics, and hence the synchronous solution is not stable to infinitesimal perturbations. (Technically, this argument shows that the synchronous solution is unstable under the $\epsilon=0$ flow. If $\epsilon>0$, the argument shows that, if the synchronous solution is stable at all, its domain of stability goes to zero as $\epsilon \rightarrow 0$.)

\section{C. Stability of the synchronous solution for indirect synapses.}

We now consider indirect synapses, and show that the synchronous solution can be stable 
in some parameter ranges. We shall show that there are two combinations of parameters that govern the stability. Furthermore, only one of those two combinations matters to the stability.

In this section, we give a precise statement of the stability result. For this result, it is necessary to make some further assumptions on the nonlinearities and parameters in (2.4), (2.6). It will be necessary to assume that

$$
f_{w}<0, \quad g_{v}>0, \text { and } g_{w}<0
$$

near the $v$-nullcline. For the main Theorem, we also assume that $f(v, w)$ is given by (2.2) with $v_{R} \leq v_{s y n}$. However, the analytical framework we develop also applies to more general nonlinearities which satisfy (3.1). This will be discussed further in Remark 11. Some technical assumptions are also required on the nonlinear function $g(v, w)$. We need to assume that $g_{v}$ is not too large near the right branches of the cubics defined by (2.11) for $s \in[0,1]$. In order to simplify the analysis that follows, we assume that $g_{v}=0$ near these right branches. The analysis in Section 3D will clearly demonstrate that the stability result remains valid if $g_{v}$ is sufficiently small, and, in fact, instabilities may arise if $g_{v}$ is too large near the right branches.

We assume that the parameters $\alpha$ and $\beta$ satisfy the conditions stated in Section 2B; that is, $\alpha$ and $\beta$ are sufficiently large, and $\frac{\alpha}{\alpha+\beta}>\theta_{\text {syn }}$. Precise conditions on how large $\alpha$ and $\beta$ must be are derived in the proof of the main Theorem. This is discussed further in Remark 12.

In order to state the main result, we need to introduce some notation. Let $a_{-}$be defined as the minimum of $-\partial g / \partial w$ over the synchronous solution in the silent phase. Note from (3.1) that $a_{-}>0$. Let $\left(w^{*}, s^{*}\right)=\left(w_{L}\left(s^{*}\right), s^{*}\right)$ be the point where the synchronous solution meets the jump-up curve, and let $\lambda=w_{L}^{\prime}\left(s^{*}\right)$ be the reciprocal slope of the jump-up curve at this point. Finally, let $a_{+}$denote the value of $g(v, w)$ evaluated on the right hand branch at the point where the synchronous solution jumps up. The main result is then the following. It holds for $\epsilon$ sufficiently small.

Theorem: Assume that the nonlinear functions and parameters in (2.4) and (2.6) satisfy the assumptions stated above. If $K<a_{-}$and $K s^{*}<a_{+} /|\lambda|$, then the synchronous solution is asymptotically stable.

Remark 1: The first condition in the Theorem is consistent with the numerical simulations of [28], who obtained synchronized solutions when the synapses recover at a rate slower than the rate at which the neurons recover in their refractory period.

Remark 2: In order to interpret the second condition in the Theorem, note that $K s^{*}$ is the rate of change of $s$ at the point at which the synchronous solution jumps. $a_{+}$is the rate of change of $w$ on the right hand branch right after the jump. Since $\lambda=d w_{L} / d s$, multiplication by $1 /|\lambda|$ transforms changes in $w$ to changes to $s$. Thus, the second condition is analogous to the compression condition that produces synchrony between relaxation oscillators coupled by fast excitation (see [21]). $|\lambda|$ may be thought of as giving 
a relationship between the time constants of inhibitory decay and recovery of the individual cells; a larger $|\lambda|$ (corresponding to a flatter curve) means that a fixed increment of decay of inhibition $(\Delta s)$ has a larger effect on the amount of recovery that a cell must undergo before reaching its (inhibition-dependent) threshold for activation.

Remark 3: The two conditions needed in the statement of the Theorem correspond to two separate cases considered in the proof of the Theorem. These two cases correspond to whether the two cells preserve their orientation (Case 1) or reverse their orientation (Case 2) on the right branch of the $s=1$ cubic after one cycle. The Theorem says that, whatever case the synchronous trajectory falls into, if both conditions hold, then the synchronous solution is stable. Note, however, that the different cases require different conditions. Case 1 requires $K<a_{-}$and Case 2 requires $K s^{*}<a_{+} /|\lambda|$. Thus, by changing a parameter that switches the system between Case 1 and Case 2, one can change which combinations of time scales and other parameters control the stability of the synchronous solution. In particular, the system can be changed between stable and unstable without changing any time constants. See Remark 5 below.

To see this more concretely, we note that if $|\lambda|$ is small, then the system will tend to be in Case 1, while if $|\lambda|$ is large, then the system will tend to be in Case 2. This will follow from analysis in the next section where we show that a necessary condition to be in Case 1 is that the first cell to jump down is the first to reach the jump-up curve. Since the leading cell lies above and to the left of the trailing cell, one expects that a more vertical jump-up curve favors the leading cell to cross this curve first. A vertical jump-up curve corresponds to small $|\lambda|$; therefore, we expect that small $|\lambda|$ favors Case 1 . If the jump-up curve is more horizontal, then the trailing cell is more likely to reach the curve first. The analysis in the next section demonstrates that this must correspond to Case 2.

Now suppose, for definiteness, that the system is in Case 2 and has a stable synchronous solution. Then the value of $a_{-}$is irrelevant and can be chosen so that $K / a_{-}$is large enough for synchrony to fail if the system were in Case 1. By lowering $|\lambda|$, the system is switched to Case 1, where the dependence on $K / a_{-}$becomes important. Similarly, if the system is in Case 1 and has a stable synchronous solution, increasing $|\lambda|$ can put it into Case 2, where the speed on the right hand branch, formerly irrelevant to stability, is now important.

Remark 4: One can give an explicit expression for $\lambda$ in terms of the conductances and reversal potentials in (2.4). In order to compute this expression, let $\Phi(v, w, s) \equiv f(v, w)-$ $g_{\text {syn }} s\left(v-v_{\text {syn }}\right)$ be the right hand side of (2.11). Plugging $v=h_{L}(w, s)$ into $(2.11)$, and differentiating with respect to both $w$ and $s$, we find that

$$
\frac{\partial h_{L}}{\partial w}=-\frac{\Phi_{w}}{\Phi_{v}} \quad \text { and } \quad \frac{\partial h_{L}}{\partial s}=-\frac{\Phi_{s}}{\Phi_{v}}
$$

Since $\frac{\partial h_{L}}{\partial w}$ becomes unbounded at the left knees, we conclude that $\Phi_{v}=0$ along the jump-up curve.

We denote the left knees by $\left(v_{L}(s), w_{L}(s)\right)$, plug this into (2.11), and then differentiate with respect to $s$ to find that

$$
0=\Phi_{v} v_{L}^{\prime}(s)+\Phi_{w} w_{L}^{\prime}(s)+\Phi_{s}=\Phi_{w} w_{L}^{\prime}(s)+\Phi_{s}
$$


Hence,

$$
\lambda=w_{L}^{\prime}\left(s^{*}\right)=-\frac{\Phi_{s}}{\Phi_{w}}=\frac{g_{s y n}\left(v_{L}\left(s^{*}\right)-v_{s y n}\right)}{f_{w}}
$$

If $f(v, w)$ is given by $(2.2)$, then $(3.3)$ becomes

$$
\lambda=-\frac{g_{s y n}\left(v_{L}\left(s^{*}\right)-v_{s y n}\right)}{g_{c}\left(v_{L}\left(s^{*}\right)-v_{R}\right)}
$$

Together with the previous remark, this expression shows that changes in reversal potentials or conductances can affect stability by changing the slope of the curve of knees.

Note that $\lambda<0$. This follows from (3.3), and the assumptions that $f_{w}<0$ and $v_{s y n}<v$ along the synchronous solution.

Remark 5: For a concrete example, we numerically solved the system given in the Appendix. We fixed all of the parameters except for $\gamma, g_{s y n}$, and $K$. The values of the other parameters are $\epsilon=.003, \alpha_{1}=.72, \alpha_{2}=2, \alpha_{3}=-.18, \alpha_{4}=0, \alpha_{5}=1, \phi=.3$, $v_{\text {syn }}=$ $-.72, \epsilon \alpha=.01, \epsilon \beta=.02, \theta_{v}=-.01$ and $\theta_{\text {syn }}=.05$

We start with the system in Case 1 and the synchronous solution is stable. For this, we let $\gamma=2, g_{\text {syn }}=.3$ and $\epsilon K=.005$. We then raise $|\lambda|$ by increasing $g_{\text {syn }}$ to 1.0. The system then goes into Case 2 and the synchronous solution becomes unstable. We then increase $a_{+}$by increasing the parameter $\gamma$ to 4.0. The synchronous solution becomes stable, as predicted by the Theorem. If we start with the original parameter values (where the system is in Case 1 and the synchronous solution is stable), then we can desynchronize the synchronous solution by increasing $K$ so that $\epsilon K=.03$.

Note that, in this case, increasing $g_{\text {syn }}$ destabilizes the system. However, the effect of increasing $g_{\text {syn }}$ is context dependent: if the system starts in Case 2 and synchrony is stable then lowering $g_{\text {syn }}$ may cause the system to go to Case 1 , where the parameters may be such that the synchronous solution is unstable. In this case, increasing $g_{\text {syn }}$ helps, rather than hurts, the synchronization. 


\section{Proof of the main theorem.}

\section{A. The return map.}

The Theorem is proved by analyzing a return map which is defined as follows. We begin both cells in the active phase with one of the cells, say cell 1, at the jump-down point. The remaining slow variables are $w_{2}, x_{1}$ and $x_{2}$; suppose that their initial positions are $w_{2}(0), x_{1}(0)$ and $x_{2}(0)$. For now, we simply assume that each $x_{i}(0)>\theta_{\text {syn }}$ and $w_{2}(0)<w_{1}(0)$ with $w_{1}(0)-w_{2}(0)$ sufficiently small. Further restrictions on the initial data are given later; see (4.2). We then follow the solution around in phase space until one of the cells returns to the jump-down point. Suppose that this happens when the slow time variable $\tau=T_{0}$. The return map is then defined as

$$
\Pi\left(w_{2}(0), x_{1}(0), x_{2}(0)\right)=\left(w_{i}\left(T_{0}\right), x_{1}\left(T_{0}\right), x_{2}\left(T_{0}\right)\right)
$$

where $i=2$ if cell 1 is the first to reach the jump-down point, and $i=1$ if cell 2 is the first to reach the jump-down point.

Of course, the return map is only well defined if the cells are able to leave their silent phase. As pointed out at the end of Section 2B, we must also assume that $\alpha$ and $\beta$ are sufficiently large with $\alpha /(\alpha+\beta)>\theta_{\text {syn }}$. This guarantees that each cell's inhibition will turn off and then turn on during each cycle.

Here we describe in more detail the sequence of events from the moment cell 1 jumps down. This will allow us to introduce some notation which will be used throughout the proof of the Theorem. Throughout this analysis, we consider the slow time variable $\tau$.

After cell $j$ jumps down to the silent phase, cell $i \neq j$ remains maximally inhibited until $x_{i}$ reaches $\theta_{\text {syn }}$. Choose $t_{i}$ so that $x_{i}=\theta_{\text {syn }}$. We assume that, at the start, the cells are sufficiently close so that the lagging cell can jump to the silent state before the inhibition on this cell starts to decrease. (That there is such an interval follows from (2.6), which implies that the value $s_{i}$ of the inhibition affecting cell $i$ stays at the maximum value $s=1$ for a finite amount of (slow) time after the other cell $j \neq i$ jumps downward. This is because $s_{i}$ does not start to decay until $x_{i}$ decays to $\theta_{s y n}$, which happens slowly.) After $t_{i}$, cell $i$ lies in the silent phase with its inhibition decreasing until its trajectory reaches the curve of knees $w_{L}(s)$. Let $T_{i}$ be the time at which the trajectory of cell $i$ reaches the curve of knees. Cell $i$ then jumps to the active phase. We assume that the cells are sufficiently close that the second cell that jumps up does so before inhibition from the first is turned on. Note that it is possible for either cell to jump up first and the cells may also reverse their order in the active phase.

We assume throughout that the cells satisfy the initial conditions needed for the definition of $\Pi$. In particular, (without loss of generality) cell 1 is at the edge $w_{R}(1)$ of the upper plateau, and cell 2 is behind it. Since cell 2 is maximally inhibited $(\mathrm{s}=1)$, the trajectory $\left(v_{2}, w_{2}\right)$ moves on a 1-dimensional path according to (2.13). We let $\delta$ denote the time it takes $w_{2}$ to get to $w_{R}(1)$ from its initial position, and let $\delta_{x}=\left|x_{1}(0)-x_{2}(0)\right|$. We assume throughout that $\delta$ and $\delta_{x}$ are sufficiently small. Finally, we suppose that one complete cycle corresponds to the slow time $\tau=T_{0}$.

We prove the Theorem by showing that if the parameters satisfy the assumptions of the Theorem, then the map $\Pi$ is a contraction. One key idea of the analysis is to separate 
the contraction into two steps. In the first, it is shown that the domain of $\Pi$ contracts under the action of $\Pi$ uniformly onto the set of solutions with synchronous initial data in which $w_{2}(0)=w_{1}(0) \equiv w_{R}(1)$ and $x_{1}(0)=x_{2}(0)$. This is a one-dimensional subset, parameterized by $x_{1}(0)$. In the second step, we show that $\Pi$, restricted to that subset, is also a uniform contraction. The two steps together give stability and the uniqueness of the synchronous solutions.

The proof is divided into cases, depending on the orders of the cells at key points in the cycle. Most of the proof describes the geometry that occurs if $t_{2}<t_{1}$, i.e. the cell that reaches the jump-down point first (cell 1) is the first to start releasing the other cell from inhibition. (Recall that $x_{i}$ depends on $v_{j}, j \neq i$.) The inequality $t_{2}<t_{1}$ is satisfied if the cells have $\left|x_{1}-x_{2}\right|$ sufficiently close at the start of the orbit. The modification for $t_{2}>t_{1}$ are given at the end of the proof.

Within the case $t_{2}<t_{1}$, there is a further distinction depending on the order at the jump-up point. In Case 1, the first cell to jump down is the first to reach the jump-up curve, and remains ahead after both cells jump up. Case 2 contains all the other possibilities. Hence, a necessary but not sufficient condition to be in Case 1 is that $T_{1}<T_{2}$. As we shall see, Case 1 corresponds to the cells preserving their orientation after one cycle, while Case 2 corresponds to the cells reversing their orientation. These subcases are only for the first contraction in the two-step scheme described above for showing that $\Pi$ is a contraction.

Before the contraction estimates, we give some preliminary results in Section 4B about the first part of the cycle. Sections $4 \mathrm{C}$ and $4 \mathrm{D}$ contain the estimates for Cases 1 and 2 for the first step of the two-step contraction proof. The estimates here are only for the $w$-variable. Section $4 \mathrm{E}$ contains the further estimates for the $x$-variable for Cases 1 and 2 . It also contains the second step of the contraction proof. Section $4 \mathrm{~F}$ gives the modification for $t_{2}>t_{1}$, and Section $4 \mathrm{G}$ contains some concluding remarks.

\section{B. Estimates relating to the jump down.}

The main result of this section is Prop 4.1, which estimates $\left|t_{2}-t_{1}-\delta\right|$ in terms of $\delta$ and $\delta_{x}$. A preliminary lemma estimates how far $x_{1}$ moves during the initial time interval of $\delta$.

Lemma 4.1: $\left|x_{1}(\delta)-x_{1}(0)\right|<\delta$.

Proof: We shall show that $x_{1}^{\prime}(\tau)<1$ for $0<\tau<\delta$, which implies the result. If $0<\tau<\delta$, both cells satisfy $v_{j}>\theta_{v}$. Using (2.15), we have

$$
x_{1}(\tau)=\frac{\alpha}{\alpha+\beta}-\left(\frac{\alpha}{\alpha+\beta}-x_{1}(0)\right) e^{-(\alpha+\beta) \tau}
$$

Hence

$$
0<x_{1}^{\prime}(\tau)=(\alpha+\beta)\left(\frac{\alpha}{\alpha+\beta}-x_{1}(0)\right) e^{-(\alpha+\beta) \tau}
$$

It is now necessary to assume that if $x_{i}(0)$ is in the domain of $\Pi$, then for any $0<m<$ $T_{0}-T_{1}$, we have

$$
\left|x_{i}(0)-\frac{\alpha}{\alpha+\beta}\right|<e^{-(\alpha+\beta) m}
$$


This is justified below. It then follows from (4.1) that $x_{1}^{\prime}(\tau)<1$ if $\alpha+\beta$ is sufficiently large.

To see why (4.2) is justified, we use (2.15) again to get

$$
x_{i}(\tau)=\frac{\alpha}{\alpha+\beta}-\left(\frac{\alpha}{\alpha+\beta}-x_{i}\left(T_{j}\right)\right) e^{-(\alpha+\beta)\left(\tau-T_{j}\right)} \quad \text { for } \quad T_{j}<\tau<T_{0}
$$

Note that $x_{i}\left(T_{j}\right)<\theta_{\text {syn }}<\frac{\alpha}{\alpha+\beta}$. Hence, $\left|\frac{\alpha}{\alpha+\beta}-x_{i}\left(T_{j}\right)\right|<1$, and (4.3) implies that

$$
\left|x_{i}\left(T_{0}\right)-\frac{\alpha}{\alpha+\beta}\right|<\left|\frac{\alpha}{\alpha+\beta}-x_{i}\left(T_{j}\right)\right| e^{-(\alpha+\beta) m}<e^{-(\alpha+\beta) m}
$$

Thus, after one cycle, the estimate (4.2) always holds, so we may assume it to begin with.

Lemma 4.2: $\left|t_{1}-t_{2}-\delta\right|<\frac{2}{\beta \theta_{s y n}}\left(\delta_{x}+\delta\right)$.

Proof: Since $v_{1}$ jumps down when $\tau=0$ and $v_{2}$ jumps down when $\tau=\delta$, it follows from (2.8) that

$$
x_{2}(\tau)=x_{2}(0) e^{-\beta \tau} \quad \text { for } \quad 0<\tau<T_{1}
$$

and

$$
x_{1}(\tau)=x_{1}(\delta) e^{-\beta(\tau-\delta)} \quad \text { for } \quad \delta<\tau<T_{2}
$$

Hence,

$$
t_{1}=\frac{1}{\beta} \ln \frac{x_{1}(\delta)}{\theta_{\text {syn }}}+\delta \quad \text { and } \quad t_{2}=\frac{1}{\beta} \ln \frac{x_{2}(0)}{\theta_{\text {syn }}}
$$

¿From (4.5), and the preceding Lemma,

$$
\begin{aligned}
\left|t_{1}-t_{2}-\delta\right| & =\left|\frac{1}{\beta} \ln \frac{x_{1}(\delta)}{x_{2}(0)}\right| \\
& =\left|\frac{1}{\beta} \ln \left(1+\frac{\left(x_{1}(\delta)-x_{1}(0)\right)+\left(x_{1}(0)-x_{2}(0)\right)}{x_{2}(0)}\right)\right| \\
& <\frac{2}{\beta} \frac{\left(\delta+\delta_{x}\right)}{x_{2}(0)}
\end{aligned}
$$

if $\delta$ and $\delta_{x}$ are sufficiently small. The result now follows since we are assuming that $x_{i}(0)>\theta_{\text {syn }}$.

\section{C. Case 1.}

We assume throughout this subsection that $t_{2}<t_{1}$. We factor the complete cycle into two maps: The first part consists of the time until cell 1 reaches the jump-up curve; that 
is, $\tau<T_{1}$. We will need to estimate the time $T_{2}-T_{1}$ until cell 2 reaches this curve. Then we consider the second half, from the jump-up of cell 1 to the time cell 1 reaches the right knee at $w=w_{R}(1)$. The hypothesis $K<a_{-}$is used only in the first half; the estimates for the second half follow with no further hypotheses.

The next result shows that, if the inhibitory decay is slow enough, the inhibition acts over the first half of the cycle to synchronize nearby orbits. For a special class of equations (including those given by $(2.2),(2.3)$ ) this synchronizing effect occurs no matter what the time scale of the inhibitory decay, but only for Case 1.

Proposition 4.1: If $K<a_{-}$, then $T_{2}-T_{1}<\rho_{1} \max \left\{\delta, \delta_{x}\right\}$ for some $\rho_{1} \in(0,1)$. (That is, there is compression in the first half of the cycle.) In the case of (2.3), in which (2.10) is valid, there is always time compression in Case 1 in this part of the cycle.

Remark 6: The last statement is relatively easy to prove, and gives an insight into how the inhibition and recovery interact in deciding stability. For the special case of (2.10), we define the $w$-metric between the two cells as the time it takes for the trailing cell 2 to reach the $w$-coordinate of the leading cell 1 . This metric remains invariant along a solution because, when (2.10) holds, the $w$-component evolves independently of $s$. Since the $w$-metric is precisely $\delta$ at the moment when cell 2 has just jumped downward, it follows that the $w$-metric remains $\delta$ throughout the silent phase. Now consider the moment when cell 1 reaches the curve $w_{L}(s)$. Cell 2 must lie to the right (since we are in Case 1) and below (since cell 1 jumped down first) cell 1 . Since $w_{L}(s)$ has negative slope, and the $w$-metric between the cells is $\delta$, it easily follows that the time it takes cell 2 to reach the curve of knees is less than $\delta$. This gives the desired compression.

The above proof uses heavily the fact that the $w$-coordinate is independent of $s$. In the more general (2.9), the $w$-coordinate is dependent on $s$; it then takes careful reasoning and estimates to show that the decay of the inhibition implies time compression; it also takes the further condition given in the hypothesis of Prop. 1.

Proof of Prop. 1: We begin by introducing some notation. Consider the image of the jump-up curve under the backward flow of (2.9) flowed until the curve reaches the position of cell 1 at time $\tau=t_{1}$. Let $t_{3}$ denote the time at which the point whose position is that of cell 2 at $\tau=t_{2}$ hits this translated curve under the forward flow. See Fig. 5 . Note that $T_{2}-T_{1}=t_{3}-t_{1}$. Hence, to show compression we need to show that $t_{3}-t_{1}<\rho_{1} \max \left\{\delta, \delta_{x}\right\}$ for some $\rho_{1} \in(0,1)$.

Let $\hat{w}_{1}(\tau) \equiv w_{1}(\tau-\delta)$. Note that $\hat{w}_{1}(\tau)$ agrees with $w_{2}(\tau)$ at the jump-down time of cell 2. That is, $\hat{w}_{1}(\delta)=w_{1}(0)=w_{2}(\delta)$. Moreover, $\hat{w}_{1}$ and $w_{2}$ both satisfy (2.9) with $s=1$ for $\delta<\tau<t_{2}$. Hence, $\hat{w}_{1}\left(t_{2}\right)=w_{2}\left(t_{2}\right)$.

Let $\lambda_{1}$ equal the (reciprocal) slope of the translated curve at cell 1 . Then (to lowest order) $\lambda_{1} \equiv \Delta W / \Delta S$, where

$$
\begin{aligned}
& \Delta W=w_{2}\left(t_{3}\right)-w_{1}\left(t_{1}\right)=w_{2}\left(t_{3}\right)-\hat{w}_{1}\left(t_{1}+\delta\right) \\
& \Delta S=s_{2}\left(t_{3}\right)-s_{1}\left(t_{1}\right)=s_{2}\left(t_{3}\right)-1
\end{aligned}
$$


Hence,

$$
\begin{aligned}
-\lambda_{1} & =\left\{\frac{w_{2}\left(t_{3}\right)-\hat{w}_{1}\left(t_{1}+\delta\right)}{1-s_{2}\left(t_{3}\right)}\right\} \\
& =\left\{\frac{w_{2}\left(t_{3}\right)-\hat{w}_{1}\left(t_{3}\right)}{1-s_{2}\left(t_{3}\right)}\right\}+\left\{\frac{\hat{w}_{1}\left(t_{3}\right)-\hat{w}_{1}\left(t_{1}+\delta\right)}{1-s_{2}\left(t_{3}\right)}\right\}
\end{aligned}
$$

The decomposition of $\lambda_{1}$ in (4.6) corresponds to the two competing effects on the positions of $w$, and hence on the slope. The first bracket on the right hand side of (4.6) is the difference in $w$ at the fixed time $t_{3}$ due to the fact that cells 1 and 2 follow different paths in the $(w, s)$ plane; since the $w$-coordinate depends on $s$ as well as $w, w_{2}\left(t_{3}\right)$ differs from $\hat{w}_{1}\left(t_{3}\right)$, even though these function agree when $\tau=t_{2}$. The second term corresponds to the difference in $w$-coordinates due to the difference between $t_{3}$ and $t_{1}+\delta$.

The proof of the proposition is now broken up into three lemmas. After stating the lemmas, we demonstrate how they are used to complete the proof of the proposition. We then present the proofs of the lemmas.

Lemma 4.3: $\lambda_{1}<0$.

Lemma 4.4: $t_{3}-t_{2}<2\left|t_{2}-t_{1}-\delta\right|$.

Lemma 4.5: If $t_{3}-t_{2}$ is $O(\delta)$ then $w_{2}\left(t_{3}\right)-\hat{w}_{1}\left(t_{3}\right)$ is $O\left(\delta^{2}\right)$.

Assuming the Lemmas 4.3 to 4.5 we may now prove Prop. 4.1. The argument is different, depending on the relative sizes of the initial differences $\delta$ and $\delta_{x}$. Hence, we have one argument for $\delta_{x}<M \delta$ for some $M$ and $\delta_{x}>M \delta$. The $M$ is arbitrary, providing it is large enough, and we pick $M=5$ for definiteness.

We must show that there is some $0<\rho<1$ such that $t_{3}-t_{1}<\rho \max \left\{\delta, \delta_{x}\right\}$. First assume that $\delta_{x}<5 \delta$. Lemmas 4.2 and 4.4 then demonstrate that $t_{3}-t_{2}$ is $O(\delta)$. ¿From Lemma 4.5, the first term on the right hand side of (4.6) is higher order in $\delta$, so we ignore it. Then, using Lemma 4.3, (4.6) implies that

$$
\hat{w}_{1}\left(t_{3}\right)-\hat{w}_{1}\left(t_{1}+\delta\right) \approx-\left(1-s_{2}\left(t_{3}\right)\right) \lambda_{1}>0
$$

Linearizing (4.7), we get $\hat{w}_{1}^{\prime}\left(t_{1}+\delta\right)\left(t_{3}-t_{1}-\delta\right) \approx-\left(1-s_{2}\left(t_{3}\right)\right) \lambda_{1}>0$ or

$$
t_{3}-t_{1}-\delta \approx \frac{-\left(1-s_{2}\left(t_{3}\right)\right) \lambda_{1}}{\hat{w}_{1}^{\prime}\left(t_{1}+\delta\right)} \equiv-c
$$

Here $c>0$ since $\hat{w}_{1}$ is monotone decreasing. For $\delta$ small enough, $0<\rho_{0}<1$ can be chosen so that $\delta-c<\rho_{0} \delta$. It then follows from (4.8) that $t_{3}-t_{1}<\rho_{0} \delta \leq \rho_{0} \max \left\{\delta, \delta_{x}\right\}$.

Now assume $\delta_{x}>5 \delta$. Since $t_{1}>t_{2}$, we have $t_{3}-t_{1}<t_{3}-t_{2}$. By Lemma 4.4, $\left|t_{3}-t_{1}\right|<2\left|t_{2}-t_{1}-\delta\right|$. To use Lemma 4.2, we now write $\left|t_{2}-t_{1}-\delta\right|<\left|t_{1}-t_{2}-\delta\right|+2 \delta$, to conclude that

$$
\left|t_{3}-t_{1}\right|<4 \delta+\frac{4\left(\delta_{x}+\delta\right)}{\beta \theta_{\text {syn }}}
$$


With $\delta<\frac{1}{5} \delta_{x}$, we have

$$
\left|t_{3}-t_{1}\right|<\left(\frac{4}{5}+\frac{5}{\beta \theta_{\text {syn }}}\right) \delta_{x}
$$

For $\beta$ sufficiently large, we can choose $\left(\frac{4}{5}+\frac{5}{\beta \theta_{s y n}}\right)<\rho_{x}<1$. Now choose $\rho_{1}$ to be the larger of $\rho_{0}$ and $\rho_{x}$.

It now remains to prove the lemmas.

Proof of Lemma 4.3: We prove this lemma by showing that the slope of the curve of knees along the synchronous solution remains negative as it is followed backwards by the flow defined by (2.9). For this we use the variational equations associated with (2.9). These equations are

$$
\begin{aligned}
\dot{W}_{i} & =-a W_{i}-b S_{i} \\
\dot{S}_{i} & =-K S_{i}
\end{aligned}
$$

where $a \equiv-\partial G_{L} / \partial w$ and $b \equiv-\partial G_{L} / \partial s$ are defined along the synchronous solution.

The evolution of this slope is governed by how $m \equiv W / S$ changes under the variational flow (4.9). This slope is governed by the equations

$$
\begin{aligned}
\dot{m} & =-b+(K-a) m \\
m(0) & =\lambda
\end{aligned}
$$

We need to prove that $m(\tau)$ remains negative for $\tau<0$. We will show that

$$
-b+(K-a) \lambda>0
$$

along the synchronous solution. This will complete the proof of the lemma, since it immediately implies that $m(\tau)<\lambda<0$ for all $\tau<0$.

We prove (4.11) by computing explicit expressions for each term on its left hand side. Since $a=-\frac{\partial G_{L}}{\partial w}=-g_{v} \frac{\partial h_{L}}{\partial w}-g_{w}$ and $b=-\frac{\partial G_{L}}{\partial s}=-g_{v} \frac{\partial h_{L}}{\partial s}$, it follows that

$$
\begin{aligned}
-b+(K-a) \lambda & =\lambda\left\{-\frac{b}{\lambda}+K-a\right\} \\
& =\lambda\left\{g_{v}\left(\frac{\partial h_{L}}{\partial w}+\frac{1}{\lambda} \frac{\partial h_{L}}{\partial s}\right)+K+g_{w}\right\}
\end{aligned}
$$

Now, $\lambda<0, g_{v}>0$, and we are assuming that $K<-g_{w}$. Hence, (4.11) follows once we show that

$$
\frac{\partial h_{L}}{\partial w}+\frac{1}{\lambda} \frac{\partial h_{L}}{\partial s} \leq 0
$$


along the synchronous solution.

We computed explicit formulas for $\frac{\partial h_{L}}{\partial w}, \frac{\partial h_{L}}{\partial s}$, and $\lambda$ in Remark 4. Let $v^{*}=v_{L}\left(s^{*}\right)$ and $p^{*}=\left(v^{*}, w^{*}, s^{*}\right)$. Then, from (3.2) and (3.3),

$$
\frac{\partial h_{L}}{\partial w}+\frac{1}{\lambda} \frac{\partial h_{L}}{\partial s}=-\frac{\Phi_{w}}{\Phi_{v}}+\frac{\Phi_{s}}{\Phi_{v}} \frac{\Phi_{w}\left(p^{*}\right)}{\Phi_{s}\left(p^{*}\right)}
$$

If $f(v, w)$ is given by $(2.2)$, then, after a little computation,

$$
\frac{\partial h_{L}}{\partial w}+\frac{1}{\lambda} \frac{\partial h_{L}}{\partial s}=\frac{g_{c}\left(v_{s y n}-v_{R}\right)\left(v^{*}-v\right)}{\Phi_{v}\left(v^{*}-v_{s y n}\right)}
$$

Inequality (4.12) now follows, because we are assuming that $v_{R} \leq v_{\text {syn }}<v^{*}$, and, on the left branch, $\Phi_{v}<0$ and $v_{\text {syn }}<v<v^{*}$.

Remark 7: The simple condition, $K<a_{-}$, for compression over the first half of the cycle depends on our assumption that $f(v, w)$ is of the form given in (2.2); in particular $f$ is linear in the variable $w$. For more general nonlinearities, (4.11) still serves as a sufficient condition for compression over the first half of the cycle. The analysis above demonstrates that each term in (4.11) - that is, $a, b$, and $\lambda$-can be expressed in terms of the parameters and nonlinear functions in the model. This will be discussed further in Remark 11 when we consider other models for inhibitory cells.

Proof of Lemma 4.4: Because of Lemma 4.3, we may conclude that $w_{2}\left(t_{3}\right)>w_{1}\left(t_{1}\right)$. (See Fig. 5.) Hence,

$$
\begin{aligned}
0 & <w_{2}\left(t_{2}\right)-w_{2}\left(t_{3}\right)<w_{2}\left(t_{2}\right)-w_{1}\left(t_{1}\right) \\
& =w_{1}\left(t_{2}-\delta\right)-w_{1}\left(t_{1}\right)
\end{aligned}
$$

Choose $m_{1}$ and $m_{2}$, both positive, so that $m_{1}<\left|w_{2}^{\prime}\right|$ and $\left|w_{1}^{\prime}\right|<m_{2}$ near the jump-down point. Together with (4.14), it follows that

$$
\left|t_{2}-t_{3}\right|<\frac{m_{2}}{m_{1}}\left|t_{2}-t_{1}-\delta\right|
$$

Note that we can assume that $m_{1}$ and $m_{2}$ are as close to each other as we please by assuming that $\delta$ and $\delta_{x}$ are sufficiently small. We may, therefore, assume that $\frac{m_{1}}{m_{2}}<2$.

Proof of Lemma 4.5: For $t_{2}<t<t_{3}, \hat{w}_{1}$ satisfies the first equation in (2.9) with $s=1$, while $w_{2}, s_{2}$ satisfy both equations in (2.9). Hence,

$$
\hat{w}_{1}\left(t_{3}\right)=\hat{w}_{1}\left(t_{2}\right)+\int_{t_{2}}^{t_{3}} G\left(\hat{w}_{1}(\tau), 1\right) d \tau
$$


and

$$
w_{2}\left(t_{3}\right)=w_{2}\left(t_{2}\right)+\int_{t_{2}}^{t_{3}} G\left(w_{2}(\tau), s_{2}(\tau)\right) d \tau
$$

We subtract these equations, use the mean value theorem, and recall that $\hat{w}_{1}\left(t_{2}\right)=w_{2}\left(t_{2}\right)$ to find that

$$
w_{2}\left(t_{3}\right)-\hat{w}_{1}\left(t_{3}\right)=\int_{t_{2}}^{t_{3}}\left\{\frac{\partial G}{\partial w}\left(w_{2}(\tau)-\hat{w}_{1}(\tau)\right)+\frac{\partial G}{\partial s}\left(s_{2}(\tau)-1\right)\right\} d \tau
$$

The partial derivatives are evaluated at some points along the synchronous solution. Choose $a_{0}$ and $b_{0}$ so that $\left|\frac{\partial G}{\partial w}\right|<a_{0}$ and $\left|\frac{\partial G}{\partial s}\right|<b_{0}$. (The bounds exist because only a compact region of the $w-s$ phase space is relevant.) Finally, note that $s_{2}(\tau)=e^{-K\left(\tau-t_{2}\right)}$. Plugging this all into (4.16), we find that

$$
\left|w_{2}\left(t_{3}\right)-\hat{w}_{1}\left(t_{3}\right)\right| \leq \frac{b_{0} K}{2}\left(t_{3}-t_{2}\right)^{2}+a_{0} \int_{t_{2}}^{t_{3}}\left|w_{2}(\tau)-\hat{w}_{1}(\tau)\right| d \tau
$$

The result now follows from Gronwall's inequality.

Remark 8: Prop. 4.1 shows that $K<a_{-} \equiv \min \left\{\left|\frac{\partial g}{\partial w}\right|\right\}$ is a sufficient condition for time compression over the first half of the cycle; it is not a necessary condition. In Remark 5 , we discussed the results of numerical simulations which demonstrate that the synchronous solution may be unstable if the condition $K<a_{-}$is not satisfied.

To complete Case 1, we now consider the behavior in the second part of the cycle. This consists of the period when the cells jump up, and the period when both cells lie in the active phase. We note that unlike the case of direct synapses, it is now possible for both cells to jump up during this first cycle. This is because once cell 1 jumps up, there is a delay (due to the $x$-variable) on the slow time scale before the inhibition $s_{2}$ jumps to $s_{2}=1$. This gives cell 2 a "window of opportunity" to reach the jump-up curve. The size of this window is determined by $\alpha+\beta$, the rate at which $x_{2}$ activates. (See (2.15).)

Proposition 4.2: When cell 1 reaches the original starting point $w_{R}(1)$, the time between the cells is less than $\rho_{1} \max \left\{\delta, \delta_{x}\right\}$.

Proof: While in the active phase, each cell lies on the right branch of the cubic determined by either $s=0$ or $s=1$. The value of $s$ switches, on the fast time scale, from 0 to 1 when the corresponding $x$-variable crosses its threshold $\theta_{\text {syn }}$. Hence, each $w_{i}$ evolves according to (2.16) with either $s=0$ or $s=1$. Eventually, both cells lie on the $s=1$ right branch (if $\alpha+\beta$ is sufficiently large). Recall that we are assuming that $g_{v}=0$ near the right branches of the cubics. Since $\frac{\partial G_{R}}{\partial s}=g_{v} \frac{\partial h_{R}}{\partial s}$, this implies that $G_{R}(w, s)$ does not depend on the variable $s$. Hence, the rate at which a cell evolves in the active phase does not depend on which right branch it lies, and we can define the distance between the cells as the time for the trailing cell to reach the $w$-coordinate of the leading cell. In 
particular, the time between two points in the active phase remains invariant. Thus, to get the time distance at the end of the right hand branch, it suffices to compute the time difference right after the jump.

For $T_{1}<t<T_{2}$, cell 1 moves up the right branch, while cell 2 still lies in the silent phase. For Case 1, we are assuming that $w_{1}\left(T_{2}\right)>w_{2}\left(T_{2}\right)$, so that the ordering of the cells on the right branch is preserved. See Fig. 6. Since $w_{1}\left(T_{1}\right)<w_{2}\left(T_{2}\right)<w_{1}\left(T_{2}\right)$, the time from $w_{2}\left(T_{2}\right)$ to $w_{1}\left(T_{2}\right)$ is less than the time from $w_{1}\left(T_{1}\right)$ to $w_{1}\left(T_{2}\right)$. This latter time is $T_{2}-T_{1}<\rho_{1} \max \left\{\delta, \delta_{1}\right\}$. Hence, the time from $w_{2}\left(T_{2}\right)$ to $w_{1}\left(T_{2}\right)$ is less than $\rho_{1} \max \left\{\delta, \delta_{1}\right\}$, which is what we needed to show.

Remark 9: A surprising aspect of the preceding analysis is that it did not use the fact that the cells are directly coupled to each other. For the compression in the first part of the cycle, it is only necessary that the cells received slowly decaying inhibition. During the second part of the cycle, the compression arose during the jump-up to the active phase. For this, it was only needed that the curve of knees has negative slope. Hence, the same analysis applies for other networks in which the cells are not directly coupled, but receive common inhibition, which turns off sufficiently slowly.

Remark 10: We have, so far, assumed that the cells are identical. However, the compression obtained over the first part of the cycle due to the slowly decaying inhibition will also help stabilize nonidentical cells, if the heterogeneity is not too large. This is illustrated in Fig. 7. In this simulation, the cells have no interactions with each other, but are provided with common inhibitory input. There is a $50 \%$ heterogeneity in the parameter $\alpha_{5}$ (see the Appendix). We compare the behavior of the cells with slowly decaying inhibition (Fig. 7A) to that with constant inhibition (Fig. 7B). Because of the heterogeneity, the population getting common inhibition starts to desynchronize. Decaying inhibition, however, acts to overcome the heterogeneity and keep the cells more synchronized, at least for a number of cycles until the inhibition wears off.

\section{D. Case 2.}

In Case 2, either cell 1 jumps up first but is behind cell 2 after it jumps up (Case 2A), or cell 2 jumps up first (Case 2B). We will show that for both of these subcases, it is cell 2 that reaches the right branch at $w=w_{R}(1)$ first. There are interesting differences between Case 1 and Case 2. In both Case $2 \mathrm{~A}$ and 2B, a different set of parameters and time scales than those for Case 1 become relevant to stability. Moreover, we saw that for Case 1 a rather complicated argument was needed to obtain compression during the silent phase. The compression due to the jump-up was straightforward. (It only required that the curve of knees has negative slope.) For Case 2, we will use a different metric during the silent phase; this will be invariant with respect to (2.9). The more delicate analysis comes into understanding the compression due to the jump-up.

Proposition 4.3: Suppose that

$$
K s^{*}<a_{+} /|\lambda|
$$

Then the time between the cells when cell 2 reaches $w_{R}(1)$ is less than $\rho_{2} \delta$ for some 
$\rho_{2} \in(0,1)$

Proof of Proposition 4.3: Let $T_{1}$ and $T_{2}$ be, as in Case 1 , the times at which the cells jump up. For Case 2A, we have that $T_{1}<T_{2}$ (cell 1 jumps up before cells 2) and $w_{1}\left(T_{2}\right)<w_{2}\left(T_{2}\right)$ (the ordering of the cells on the right branch is reversed). This is illustrated in Fig. 8. Let $\delta_{1}$ be the (time) distance on the right branch from $w_{1}\left(T_{2}\right)$ to $w_{2}\left(T_{2}\right)$. Recall that this metric does not depend on which right branch the cells lie. We need to show that $\delta_{1}<\rho_{2} \delta$ for some $\rho_{2} \in(0,1)$. Since we are computing the stability of the synchronized solution, we may assume that $w_{1}\left(T_{1}\right)$ and $w_{2}\left(T_{2}\right)$ are arbitrarily close. This enables us to linearize the calculation around the quantities associated with the synchronized solution. We use the notation $\approx$ to mean that higher order terms in a Taylor expansion have been omitted.

We start with a characterization of $\lambda$ in terms of spatial quantities. $\lambda$ is $\Delta W / \Delta S$ for two nearby points in the curve $w_{L}(s):\left(w_{1}\left(T_{1}\right), s_{1}\left(T_{1}\right)\right)$ and $\left(w_{2}\left(T_{2}\right), s_{2}\left(T_{2}\right)\right)$. Thus,

$$
\lambda \approx \frac{w_{2}\left(T_{2}\right)-w_{1}\left(T_{1}\right)}{s_{2}\left(T_{2}\right)-s_{1}\left(T_{1}\right)}=\frac{\left(w_{2}\left(T_{2}\right)-w_{1}\left(T_{2}\right)\right)+\left(w_{1}\left(T_{2}\right)-w_{1}\left(T_{1}\right)\right)}{\left.\left(s_{2}\left(T_{2}\right)-s_{2}\left(T_{1}\right)\right)+\left(s_{2}\left(T_{1}\right)\right)-s_{1}\left(T_{1}\right)\right)}
$$

We next express each term on the right hand side of (4.11) as a time quantity. The time on the right branch from $w_{1}\left(T_{2}\right)$ to $w_{2}\left(T_{2}\right)$ is, by definition, $\delta_{1}$, and the time from $w_{1}\left(T_{1}\right)$ to $w_{1}\left(T_{2}\right)$ is $T_{2}-T_{1}$. Since the rate on the right branch is approximately $a_{+}$,

$$
\left(w_{2}\left(T_{2}\right)-w_{1}\left(T_{2}\right)\right)+\left(w_{1}\left(T_{2}\right)-w_{1}\left(T_{1}\right)\right) \approx a_{+}\left(\delta_{1}+T_{2}-T_{1}\right)
$$

The time in the silent phase from $s_{2}\left(T_{1}\right)$ to $s_{2}\left(T_{2}\right)$ is $T_{2}-T_{1}$. Since each $s_{i}$ evolves at approximately the rate $-K s^{*}$,

$$
s_{2}\left(T_{1}\right)-s_{2}\left(T_{2}\right) \approx K s^{*}\left(T_{2}-T_{1}\right)
$$

It remains to express $s_{1}\left(T_{1}\right)-s_{2}\left(T_{1}\right)$ as a time quantity. Note that the differential equation for each $s_{i}$ does not depend on $w_{i}$. Hence, the time it takes $s$ to go from its value at cell 1 to its value at cell 2 remains invariant during the silent phase, and does not depend on the particular value of $w$. We denote this time as the $s$-metric between the two cells. Note that when $\tau=t_{1}$, the $s$-metric between the cells is precisely $t_{1}-t_{2}$. Moreover, the rate at which $s$ evolves when $t=T_{1}$ is approximately $-K s^{*}$. Hence,

$$
s_{1}\left(T_{1}\right)-s_{2}\left(T_{1}\right) \approx K s^{*}\left(t_{1}-t_{2}\right)
$$

Combining (4.18) and (4.19a,b,c) we find that

$$
-\lambda \approx \frac{a_{+}\left(\delta_{1}+T_{2}-T_{1}\right)}{K s^{*}\left(\left(t_{1}-t_{2}\right)+T_{2}-T_{1}\right)}
$$


or

$$
\begin{aligned}
\delta_{1} & \approx \frac{|\lambda| K s^{*}}{a_{+}}\left(t_{1}-t_{2}\right)+\left(\frac{|\lambda| K s^{*}}{a_{+}}-1\right)\left(T_{2}-T_{1}\right) \\
& \leq \frac{|\lambda| K s^{*}}{a_{+}}\left(t_{1}-t_{2}\right)
\end{aligned}
$$

if $\frac{|\lambda| K s^{*}}{a_{+}}<1$. Now Lemma 4.2 implies that

$$
\begin{aligned}
\delta_{1} & \leq \frac{|\lambda| K s^{*}}{a_{+}} \delta+\frac{|\lambda| K s^{*}}{a_{+}} \frac{2}{\beta \theta_{\text {syn }}}\left(\delta+\delta_{x}\right) \\
& \leq \rho_{2} \max \left\{\delta, \delta_{x}\right\}
\end{aligned}
$$

for some $\rho_{2} \in(0,1)$ if $\frac{|\lambda| K s^{*}}{a_{+}}<1$ and $\beta$ is sufficiently large.

Finally, consider Case 2B, in which cell 2 jumps before cell 1 ; that is, $T_{2}<T_{1}$. For $T_{2}<\tau<T_{1}$, cell 2 moves up the right branch, while cell 1 still lies in the silent phase. See Fig. 9. Moreover, for $T_{2}<\tau<T_{1}, w_{1}\left(T_{1}\right)<w_{2}\left(T_{2}\right)<w_{2}(\tau)$; that is, the cells can not reverse their ordering after they jump up. We need to prove that if $\delta_{2}$ is the time on the right branch from $w_{1}\left(T_{1}\right)$ to $w_{2}\left(T_{1}\right)$, then $\delta_{2}<\rho_{2} \delta$.

The proof is very similar to that for Case $2 \mathrm{~A}$. We begin by writing $\lambda$ in terms of spatial quantities; however, since $T_{2}$ is now less than $T_{1}$, we break up $\lambda$ somewhat differently:

$$
\lambda=\frac{w_{2}\left(T_{2}\right)-w_{1}\left(T_{1}\right)}{s_{2}\left(T_{2}\right)-s_{1}\left(T_{1}\right)}=\frac{\left(w_{2}\left(T_{1}\right)-w_{1}\left(T_{1}\right)\right)-\left(w_{2}\left(T_{1}\right)-w_{2}\left(T_{2}\right)\right)}{\left(s_{2}\left(T_{2}\right)-s_{1}\left(T_{2}\right)\right)-\left(s_{1}\left(T_{1}\right)-s_{1}\left(T_{2}\right)\right)}
$$

We next express each of these terms as a time quantity. As before,

$$
\left(w_{2}\left(T_{1}\right)-w_{1}\left(T_{1}\right)\right)-\left(w_{2}\left(T_{1}\right)-w_{2}\left(T_{2}\right)\right) \approx a_{+}\left(\delta_{2}-\left(T_{2}-T_{1}\right)\right)
$$

Moreover,

$$
s_{1}\left(T_{2}\right)-s_{1}\left(T_{1}\right) \approx K s^{*}\left(T_{2}-T_{1}\right)
$$

and, using that the $s$-metric is invariant,

$$
s_{2}\left(T_{2}\right)-s_{1}\left(T_{2}\right) \approx K s^{*}\left(t_{1}-t_{2}\right)
$$

Combining (4.20) with (4.21a,b,c) yields

$$
-\lambda \approx \frac{a_{+}\left(\delta_{2}-\left(T_{2}-T_{1}\right)\right)}{K s^{*}\left(\left(t_{1}-t_{2}\right)-\left(T_{2}-T_{1}\right)\right)}
$$

The proof now proceeds as before. 


\section{E. Contraction of the $x$-variables.}

To produce a contraction of the open set of initial conditions onto a one-dimensional subset parameterized by $x_{1}(0)$, it remains to show that $\left|x_{1}\left(T_{0}\right)-x_{2}\left(T_{0}\right)\right|<\max \left\{\delta, \delta_{x}\right\} / 2$. This is done in two steps. First we estimate $\left|x_{1}\left(T_{1}\right)-x_{2}\left(T_{2}\right)\right|$. For this estimate, note that $\left|T_{1}-T_{2}\right|<2 \max \left\{\delta, \delta_{x}\right\}$. For Case 1 , this was proved in Proposition 1 and this Proposition also applies for Case $2 \mathrm{~A}$. For Case 2B, we use that the $s$-metric is invariant under the flow in the silent phase. This implies that the time it takes $s$ to flow from $s_{1}\left(T_{2}\right)$ to $s_{2}\left(T_{2}\right)$ is precisely the time it takes $s$ to flow from $s_{1}\left(t_{1}\right)$ to $s_{2}\left(t_{1}\right)$, which is $t_{1}-t_{2}$. Now, in Case 2B, cell 1 reaches the curve of knees before $s_{1}$ reaches $s_{2}\left(T_{2}\right)$; that is, $s_{1}\left(T_{1}\right)>s_{2}\left(T_{2}\right)$. It follows that $0<T_{1}-T_{2}<t_{1}-t_{2}$. This, together with Lemma 4.2 , implies that $\left|T_{1}-T_{2}\right|<2 \max \left\{\delta, \delta_{x}\right\}$ if the parameter $\beta$ is sufficiently large.

It follows from (4.4) that $x_{1}\left(T_{1}\right)=x_{1}(\delta) e^{-\beta\left(T_{1}-\delta\right)}$ and $x_{2}\left(T_{2}\right)=x_{2}(0) e^{-\beta T_{2}}$. Together with the mean value theorem and Lemma 4.1, this implies that

$$
\begin{aligned}
\left|x_{1}\left(T_{1}\right)-x_{2}\left(T_{2}\right)\right| & <x_{2}(0)\left|e^{-\beta\left(T_{1}-\delta\right)}-e^{-\beta T_{2}}\right|+\left|x_{1}(\delta)-x_{2}(0)\right| e^{-\beta\left(T_{1}-\delta\right)} \\
& <2 \beta x_{2}(0) e^{-\beta T_{2}}\left|T_{2}-T_{1}-\delta\right|+\left(\delta+\delta_{x}\right) e^{-\beta\left(T_{1}-\delta\right)} \\
& <\frac{1}{2} \max \left\{\delta, \delta_{x}\right\}
\end{aligned}
$$

if $\beta$ is sufficiently large.

The analysis for the second part of the cycle is quite similar. We use (4.3) and the mean value theorem to conclude that if $\alpha+\beta$ is sufficiently large, then

$$
\begin{aligned}
\left|x_{1}\left(T_{0}\right)-x_{2}\left(T_{0}\right)\right|< & \left(\frac{\alpha}{\alpha+\beta}-x_{1}\left(T_{1}\right)\right) \mid e^{-(\alpha+\beta)\left(T_{0}-T_{1}\right)}-e^{(\alpha+\beta)\left(T_{0}-T_{2}\right) \mid} \\
& +\left|x_{1}\left(T_{1}\right)-x_{2}\left(T_{2}\right)\right| e^{-(\alpha+\beta)\left(T_{0}-T_{2}\right)} \\
<2( & \left.\frac{\alpha}{\alpha+\beta}-x_{1}\left(T_{1}\right)\right)(\alpha+\beta) e^{-(\alpha+\beta)\left(T_{0}-T_{1}\right)}\left|T_{1}-T_{2}\right| \\
& \quad+2 \max \left\{\delta, \delta_{x}\right\} e^{-(\alpha+\beta)\left(T_{0}-T_{2}\right)} \\
< & \max \left\{\delta, \delta_{x}\right\} / 2
\end{aligned}
$$

To show that the one-dimensional subset parameterized by $x_{1}(0)$ itself contracts down to a unique orbit, we use a small modification of the above estimates. We are now assuming that cells 1 and 2 start with identical conditions, so $\delta=\delta_{x}=0$. We consider two pairs of cells $x_{1}, x_{2}$ and $\bar{x}_{1}, \bar{x}_{2}$ with $x_{1}=x_{2}$ and $\bar{x}_{1}=\bar{x}_{2}$. Let $T_{0}$ and $\bar{T}_{0}$ be the times when these cells complete one cycle. We show that $\left|x_{1}\left(T_{0}\right)-\bar{x}_{1}\left(\bar{T}_{0}\right)\right|<\rho\left|x_{1}(0)-\bar{x}_{1}(0)\right|$ for some $0<\rho<1$. 
The first inequality of (4.22) is as before, with $x_{2}$ replaced by $\bar{x}_{1}$ and $T_{2}$ by $\bar{T}_{1}$, where $\bar{T}_{1}$ is the time for the pair $\bar{x}_{1}, \bar{x}_{2}$ to reach the jump-up curve. One finds that

$$
\begin{gathered}
\left|x_{1}\left(T_{1}\right)-\bar{x}_{1}\left(\bar{T}_{1}\right)\right| \leq \bar{x}_{1}(0)\left|e^{-\beta\left(\bar{T}_{1}-T_{1}\right)}-e^{-\beta \bar{T}_{1}}\right|+\left|x_{1}(0)-\bar{x}_{1}(0)\right| e^{-\beta T_{1}} \\
\leq 2 \beta \bar{x}_{1}(0) e^{-\beta \bar{T}_{1}}\left|\bar{T}_{1}-T_{1}\right|+\left|x_{1}(0)-\bar{x}_{1}(0)\right| e^{-\beta T_{1}}
\end{gathered}
$$

It is now necessary to estimate $\left|\bar{T}_{1}-T_{1}\right|$ in terms of $\left|x_{1}(0)-\bar{x}_{1}(0)\right|$. An argument almost identical to that given above shows that $\left|\bar{T}_{1}-T_{1}\right|<2\left|x_{1}(0)-\bar{x}_{1}(0)\right|$. Plugging this into (4.24) and assuming that $\beta$ is sufficiently large, we get

$$
\left|x_{1}\left(T_{1}\right)-\bar{x}_{1}\left(\bar{T}_{1}\right)\right|<\frac{1}{2}\left|x_{1}(0)-\bar{x}_{1}(0)\right|
$$

Using this with the analogue of (4.23), we get the desired contraction.

4F. $t_{1}<t_{2}$.

Now assume that $t_{1}<t_{2}$. Then in the silent phase, cell 1 leaves the curve $s=1$ of maximal inhibition before cell 2 does. Once again there are two cases to consider and these correspond to the two cases considered in the previous section. It will be more convenient, however, to describe these cases somewhat differently than before. Our description will be in terms of whether $t_{2}-t_{1} \leq \delta$ or $t_{2}-t_{1}>\delta$. We begin with the following Lemma which relates $\delta$ and $\delta_{x}$.

Lemma 4.6: If $t_{1}<t_{2}$, then $\delta<\frac{2}{\beta \theta_{\text {syn }}-2} \delta_{x}$.

Proof: It follows from (4.5) and a calculation which is given in the proof of Lemma 4.2 that if $t_{1}<t_{2}$, then

$$
\delta<\left|\frac{1}{\beta} \ln \frac{x_{1}(\delta)}{x_{2}(0)}\right|<\frac{2}{\beta} \frac{\left(\delta+\delta_{x}\right)}{\theta_{\text {syn }}}
$$

We then solve for $\delta$ and the proof is complete.

Now assume that $t_{2}-t_{1} \leq \delta$. Then $w_{1}\left(t_{1}\right) \leq w_{2}\left(t_{2}\right)$; see Figure 10A. This is because for $\delta<\tau<t_{2}$, each $w_{i}^{\prime}(\tau)<0$ and $w_{2}(\tau)=w_{1}(\tau-\delta)$. Hence, if $t_{2}-t_{1} \leq \delta$, then $w_{2}\left(t_{2}\right)=$ $w_{1}\left(t_{2}-\delta\right) \geq w_{1}\left(t_{1}\right)$. It follows that while in the silent phase, the trajectory corresponding to cell 2 must remain to the 'right' (larger $w$-values) of the trajectory corresponding to cell 1 . Hence, the points at which these cells jump up satisfy $w_{1}\left(T_{1}\right)<w_{2}\left(T_{2}\right)$ and $s_{1}\left(T_{1}\right)>s_{2}\left(T_{2}\right)$. The assumption $t_{1}<t_{2}$ implies that $s_{1}\left(t_{2}\right)<1=s_{2}\left(t_{2}\right)$. Since $s_{1}$ and $s_{2}$ both satisfy the same equation, it follows that $s_{1}(\tau)<s_{2}(\tau)$ for all $\tau \in\left(t_{2}, T_{1}\right)$. We may now conclude that $T_{1}<T_{2}$.

We next show that $T_{2}-T_{1}<\delta_{x} / 2$. This is proved in a way very similar to that of Proposition 1 in the previous section. We consider the image of the jump-up curve under the backward flow of (2.9) flowed until the curve reaches the position of cell 1 at time 
$\tau=t_{1}$. Let $t_{3}$ denote the time at which the point whose position is that of cell 2 at $\tau=t_{2}$ hits this translated curve under the forward flow. Note that

$$
T_{2}-T_{1}=t_{3}-t_{1}=\left(t_{3}-t_{2}\right)+\left(t_{2}-t_{1}\right)
$$

Lemma 4.6 implies that $t_{2}-t_{1} \leq \delta<\delta_{x} / 4$ if $\beta$ is sufficiently large. We estimate $t_{3}-t_{2}$ exactly as we did in Lemma 4.4. The inequality (4.15), together with Lemmas 4.2 and 4.6, implies that $\left|t_{3}-t_{2}\right|<\delta_{x} / 4$ if $\beta$ is sufficiently large. We have therefore shown that $T_{2}-T_{1}<\delta_{x} / 2$.

We next estimate $\left|w_{1}\left(T_{0}\right)-w_{2}\left(T_{0}\right)\right|$. If cell 1 is ahead of cell 2 after they jump up, then just as in the proof of Proposition 2, we have that the time on the right branch from cell 2 to cell 1 is less than $T_{2}-T_{1}<\delta_{x} / 2$. Now suppose that cell 1 is behind cell 2 after they both jump up. This is very similar to Case $2 \mathrm{~A}$ in the previous section. Proceeding exactly as before, we write $\lambda$ as in (4.18) and then express each term in (4.18) as a time quantity. If $\delta_{1}$ is the time on the right branch from $w_{2}\left(T_{2}\right)$ to $w_{1}\left(T_{2}\right)$, then the conclusion of this computation is that if $K s^{*}<a_{+} /|\lambda|$, then $\delta_{1}<t_{2}-t_{1}$. We are assuming that $t_{2}-t_{1}<\delta$ and Lemma 4.6 implies that $\delta<\delta_{x} / 4$. Hence, the time on the right branch between the cells must be less than $\delta_{x} / 2$.

It remains to consider the case $t_{2}-t_{1}>\delta$. Then during the silent phase, the trajectory corresponding to cell 2 lies to the 'left' of that corresponding to cell 1. See Figure 10B. Hence, the points at which the cells jump up satisfy $w_{2}\left(T_{2}\right)<w_{1}\left(T_{1}\right)$ and $s_{2}\left(T_{2}\right)>s_{1}\left(T_{1}\right)$. We claim that $T_{1}<T_{2}$. To prove this, we consider the image of the jump-up curve under the backward flow (2.9) until this curve reaches the position of cell 1 at time $\tau=t_{2}$. Lemma 4.3 implies that if $\hat{\lambda}$ is the slope of this translated curve, then $\hat{\lambda}<0$ as shown in Figure 10B. We next show that cell 2 lies above this translated line segment at time $t_{2}$. We start by noting that $w_{1}\left(t_{1}\right) \leq w_{2}\left(t_{1}\right)$, since cell 1 falls down first. Since $\hat{\lambda}<0$, to have cell 2 be above the translated line, we must have

$$
w_{1}\left(t_{2}\right)-w_{2}\left(t_{2}\right) \leq \hat{\lambda}\left(s_{1}\left(t_{2}\right)-s_{2}\left(t_{2}\right)\right)
$$

(See Fig. 10B.) Thus the worst initial conditions at $t_{1}$ are $w_{1}\left(t_{1}\right)=w_{2}\left(t_{1}\right)$. For such initial conditions, estimates similar to those of Lemma 4.5 imply that $w_{2}\left(t_{2}\right)-w_{1}\left(t_{2}\right)$ is order $\left(t_{2}-t_{1}\right)^{2}$. Furthermore, $s_{2}\left(t_{2}\right)-s_{1}\left(t_{2}\right)=s_{1}\left(t_{1}\right)-s_{1}\left(t_{2}\right)$ is order $\left(t_{2}-t_{1}\right)$. Since $\hat{\lambda}$ is order one, (4.25) is satisfied. It now follows that cell 1 reaches the jump-up curve before cell 2 ; i.e., $T_{1}<T_{2}$.

We then proceed exactly as in Case $2 \mathrm{~B}$ in the previous section. That is, we express $\lambda$ as in (4.20) and then write each term as a time quantity. Once again, we conclude that if $K s^{*}<a_{+} /|\lambda|$, then the time on the right branch between the cells is less than $t_{2}-t_{1}<\delta_{x} / 2$.

We must also verify that $\left|x_{1}\left(T_{0}\right)-x_{2}\left(T_{0}\right)\right|<\delta_{x} / 2$. There is, however, no difference between the arguments needed here and the corresponding ones given in Section $4 \mathrm{E}$.

\section{G. Concluding Remarks.}

Remark 11: The techniques used to prove the Theorem apply to other models besides those which satisfy its specific hypothesis. For example, $w$ may represent the concentration 
of a calcium current instead of the activation of a potassium current [28]. In [28] each cell is modeled by an equation of the form (2.1); however, the left and right branches are increasing functions and the $w$-nullcline is a decreasing function. Moreover instead of (3.1), $f$ and $g$ satisfy $f_{w}<0, g_{v}<0$, and $g_{w}<0$. If $f(v, w)$ is of the form given by $(2.2)$, then the Theorem applies directly to these other models. The proof is basically the same; there are still two cases to consider. The only minor difference is that during the silent phase $w$ increases to a curve of knees with positive slope, while before $w$ decreased to a curve of knees with negative slope.

If $f(v, w)$ is not given by $(2.2)$, then the techniques used to prove the Theorem still apply; however the precise stability conditions may change. The precise form of $f$ was used only in Case 1, where we used that $f(v, w)$, as given by $(2.2)$, is linear in $w$. If $f$ is not linear in $w$, then (4.4) still gives a sufficient condition for compression in the silent phase. Each quantity in (4.4) - that is, $a, b$ and $\lambda$-can be written in terms of the nonlinear functions $f$ and $g$ as was done in the paragraph following (4.4). This then leads to a sufficient condition for stability in terms of the nonlinear functions in the model.

Remark 12: Recall that the synchronous solution can be stable only when the model includes indirect synapses. The $x$-variable provides a delay on the slow time scale from when one cell jumps up until inhibition can prevent the other cell from firing. The size of this "window of opportunity" is determined by $\alpha+\beta$, the rate at which the $x$-variable activates; if $\alpha+\beta$ is large, then the window is small. This result is consistent with those of [5] and [26] who obtain stable synchronous solutions for integrate and fire type models. They demonstrate that for those models, the synchronous solution is stable if the rate at which the inhibition turns on is sufficiently slow. This rate corresponds to $\alpha+\beta$ in our model.

In our analysis, however, we need to assume that $\alpha$ and $\beta$ are not too small. This is because if they are too small, then the inhibition will not turn on or turn off during each cycle. For this we also need that $\frac{\alpha}{\alpha+\beta}>\theta_{\text {syn }}$. (See the discussion in Section 2B.) These last conditions are not consistent with those in [3] and [26]. This inconsistency can be understood by considering the differences in the way the coupling between cells is modeled. In the integrate and fire models, the inhibition to a cell is automatically turned on whenever the other cell crosses threshold. In our model, however, the inhibition to a cell (say $j$ ) is turned on only if the variable $x_{j}$ crosses threshold. $x_{j}$ is able to cross the threshold only if the other cell remains in its active phase for a sufficiently long period of time. This time can be made arbitrarily small if the rate $\alpha+\beta$ at which $x_{j}$ activates is sufficiently large.

\section{Nonsynchronous solutions.}

The network of two mutually inhibitory cells can display other behaviors. We will not give rigorous conditions for the existence and stability of these other solutions; instead, we give simulations of the other solutions, and a general description of the parameter ranges in which they are expected. The heuristic explanations we give are based on the techniques developed in the previous section. For all of the examples, we consider direct synapses, although the analysis for indirect synapses is very similar. In the numerical simulations, we consider the system given in the Appendix. Unless stated otherwise, parameters values 
common to each of these examples are: $\epsilon=.002, \alpha_{1}=.47, \alpha_{2}=1, \alpha_{3}=-.19, \alpha_{4}=$ $-.4, \alpha_{5}=1 ., \gamma=1, \theta_{\text {syn }}=-.01$ and $\phi=1$.

We start with the antiphase solution. Such a solution is shown in Fig. 11B. The antiphase solution is the most well-known solution for a pair of mutually inhibitory oscillators, expected when the inhibition decays at a rate faster than the recovery of the oscillator $\left(K / a_{-}\right.$large). (See, for example, [20, 23, 28].) Such a solution can exist even if the cells are excitable, rather than oscillatory, via the mechanism of "post-inhibitory rebound" [4]. (We note that this does not require any special pacemaker currents, such as hyperpolarization activated inward currents.) Though $K / a_{-}$small favors stability of the synchronous solution and $K / a_{-}$large favors the existence of a stable antiphase solution, there is a parameter range in which both solutions are stable. For Fig. $11 \mathrm{~B}, K=5$.

One can describe the evolution of the antiphase solution in phase space in a way similar to the description of the synchronous solution given earlier. In Fig. 12, we illustrate the projection of an antiphase solution onto the w, s plane. We choose the initial (slow) time to be when both cells lie in the silent phase, and cell 1 has just jumped down from the active phase. This implies that $s_{2}(0)=1$. Both cells then evolve in the silent phase until cell 2 reaches the jump-up curve. We denote this time to be $\tau=\tau_{1}$. At this time, $s_{1}$ jumps up to the line $s \equiv 1$. Cell 2 then evolves in the active phase; we illustrate the projection of cell 2's trajectory during the active phase with a dotted curve in Fig. 12. Note that $s_{2}(\tau)$ still satisfies $s_{2}^{\prime}=-K s_{2}$; hence, it keeps decreasing while cell 2 is active. During this time, cell 1 lies in the silent phase with $s_{1}=1$. This continues until cell 2 reaches the jump-down curve $w_{R}(s)$. We denote this time as $\tau_{2}$. Cell 2 then jumps down and this completes one-half of a complete cycle. For this to be an antiphase solution, we must have that $w_{1}\left(\tau_{2}\right)=w_{2}(0)$ and $s_{2}\left(\tau_{2}\right)=s_{1}(0)$. Rigorous results related to the existence and stability of antiphase solutions for systems with slow inhibitory coupling are given in [23].

Another kind of nonsynchronous solution obtained in this system was referred to in the introduction as "suppressed solutions", and is shown in Fig. 11A. These are ones in which one cell remains quiet while the other oscillates. They occur in the same parameter range as the stable synchronous solutions, i.e. $K / a_{-}$small. The behavior of these solutions is easy to understand: if the inhibition decays slowly enough, the leading cell can recover and burst again before the inhibition from its previous burst wears off enough to allow the other cell to fire. This type of solution cannot exist if the cells are excitable rather than oscillatory, since there is no input from the quiet cell to drive the active one. On the other hand, the cells must also be excitable for some fixed levels of inhibition; i.e. some $s \in(0,1]$. If this is not the case, then the $w$-coordinate of the suppressed cell must keep decreasing until that cell eventually reaches the jump-up curve and fires. For Fig. 11A, $\alpha_{3}=-.15$ and $K=.5$.

If the synaptic inhibition decays at a rate comparable to the recovery of the cell, complex hybrid solutions can occur, in which one cell is suppressed for several cycles, while the other fires, and then fires while the other is suppressed. Examples of these are shown in Figures 13 and 14. In Fig. 13, cell 1 fires three times, while cell 2 is suppressed, and then cell 2 fires three times, while cell 1 is suppressed. In Fig. 14, cell 1 fires twice keeping cell 2 suppressed, and then cell 2 fires only once. In both of these examples, each cell, without any input, is excitable, but is oscillatory for some intermediate levels of inhibition. Hence, if $K / a_{-}$is sufficiently small, a cell can fire a number of times while the other cell is 
suppressed. The inhibition of the spiking cell must eventually wear off, so that cell can no longer fire. This then allows the inhibition of the suppressed cell to wear off to the point where it can now fire. The roles of the two cells are then reversed. The rapid, subthreshold oscillations shown in Fig. 13 arise because the stable fixed point of the full system has complex eigenvalues. For both Fig. 13 and Fig. $14, K=.3$. For Fig. $14, \phi=.01$.

The synchronous solution exists stably in parameter regimes in which one or more of the above nonsynchronous solutions is also stable. Thus the choice of solution depends on the initial condition. The basin of attraction of the synchronous solution depends mainly on the delay of the onset of the inhibition. In order for a trajectory to be in the domain of attraction of the synchronous solution, the lagging cell must be activated before the inhibition from the leading cell delays it. As the onset time of inhibition decreases, the domain of stability of the synchronous state vanishes, but the nonsynchronous solutions remain.

\section{Discussion.}

\section{A. Related Work.}

Related papers that deal with two or more oscillators coupled only through inhibition are $[1-8,15,18,19,20,25-30,33]$. In $[5,26,28]$, it is shown that slow inhibition can lead to synchrony of oscillators. The analytical results of van Vreeswijk, et al. [26] are for integrate and fire models of neurons, and synapses having the "alpha function" form $g \alpha^{2} t e^{-\alpha t}$. Gerstner, et al. [5] also use model neurons with a very short spiking time. The analytical results presented here use a more biophysically based neuronal model with more degrees of freedom, and a model of a synapse that allows one to sort out the different effects of slow onset and slow offset (more in the spirit of the simulations of the biophysical models in [28]). The current work shows that the slow onset acts to provide a window of opportunity for the firing of the other oscillator right after one has fired, and hence affects mainly the domain of attraction of the synchronous solution (see Remark 12). The slow offset has a more subtle affect. For Case 1, it pulls trajectories closer to one another during the silent phase. For Case 2, it prevents instabilities from arising during the jump up to the active phase.

The work of van Vreeswijk, et al. [26] and Gerstner, et al. [5] shows both similarities and differences with ours. Using synapses with instantaneous onset, [26] obtains instabilities. This is consistent with our result that the time of onset provides a window of opportunity for synchrony. Their analytical work using alpha function synapses comes to conclusions different in detail from ours. They conclude that synchrony is always stable; for slow synapses, this is the only stable solution and for larger alpha, the antiphase state is also stable. Similarly, the work of Gerstner, et al. [5] shows that the conditions for stability of the completely synchronous solution holds except for extreme cases, in which inhibition sets in very slowly. Thus, as in [26], the decay time of inhibition does not play a central role in the stability of the synchronized solution. Furthermore, though the rise time of the inhibition is not zero in our models, it can be considerably shorter than the width of a burst (or broad action potential), and still allow synchrony. (See [26] for discussion suggesting the opposite.)

Recently Chow and Ritt [34] have performed a stability analysis on the synchronous 
solution for an integrate and fire neuron but using synapses with a double exponential time course $e^{-\beta t}-e^{-\alpha t}$ or with an exponential with delay $e^{-\beta(t-\delta)}$. In this work, synchrony is stable provided that the delay is not zero or the rise time is not infinitely fast. For a short finite rise time (delay) both the synchronous and anti-synchronous solutions are stable. As in the current work, the rise time controls the domain of attraction of the respective fixed points. For rise times longer than a critical time, the anti-synchronous solution can vanish if the decay time also exceeds a critical value. As in $[5,26]$, there is no condition that the inhibitory decay time be large enough.

In our model (as in [28]), synchrony can be unstable if the decay time of the inhibitory synapse is too short. We note that our conditions $K<a_{-}$, that the inhibitory decay time be adequately long relative to the cell recovery time, is not needed when using the simple equation (2.3), for which the equations in the silent phase are the linear equations (2.10). The integrate and fire equation of $[26,34]$ is also linear between spikes. We conjecture that a condition analogous to $K<a_{-}$will be found for more general spiking models, possibly with a dependence of the voltage threshold on the level of inhibition.

Our analysis also shows that other parameters not in the integrate and fire model, such as the time on the excited branch and the relative size of the synaptic conductance and the potassium conductance, can affect the outcome. (This was also seen in the simulations of $[8,28])$. Indeed, the analysis shows that the parameters interact in subtle ways. For example, increasing the strength of the synapse pushes the trajectory from Case 1 (in which the oscillator first to jump down is also the first to jump up) to Case $2 \mathrm{~B}$ (with the opposite ordering). In Case 2 (but not Case 1), parameters such as the length of the activated portion of the cycle are relevant; thus the length of the activated state might or might not play a role in the stability depending on whether the synapse is strong enough. This helps to explain, for example, why, in the presence of slow decay of inhibition, making the fast inhibition shunting (i.e. making $v_{s y n}$ less negative, so $\left(v-v_{\text {syn }}\right)$ is smaller) can cause the synchronous solution to be stable (see [8]). Suppose we choose parameters so that Case 2 is relevant, and $a_{+}$is sufficiently small so that the synchronous solution is unstable. Making $v_{\text {syn }}$ less negative has the effect of decreasing $|\lambda|$; this can ensure that Case 1 becomes the relevant one. If $a_{-}$(which was irrelevant before) is sufficiently large, then the synchronous solution will become stable.

The analytical results presented here are for variations of the Morris-Lecar oscillator, but the geometric techniques from which the results are derived are applicable to general oscillators having different time scales, including the ones used by Wang and Rinzel [28] (see Remark 11). The heuristic explanations given in [28] are in terms of the properties of specific channels. Our work shows how similar phenomena can occur in a more general setting that does not make use of the special currents $I_{T}$ or $I_{h}$. In particular, our work reinforces the conclusion of [28] that the decay time of the inhibition is important to the synchrony, and shows that this does not require the post-inhibitory rebound associated with the currents $I_{h}$ and $I_{T}$.

For a pair of cells, Wang and Rinzel [28] varied the synaptic decay time and the reversal potential for the synapse, and obtained stable synchrony, antiphase, no activity and asymmetric steady states. They did not observe the solutions with one suppressed permanently or transiently, as we did. For larger networks, they did observe dynamics in which some cells suppressed others. The current work shows that it does not require large networks 
to get that effect. These more "exotic" solutions can be constructed by similar geometric means, but this is outside the scope of this paper.

\section{B. Locus of Control.}

Previous work on mutually inhibitory neurons emphasized the distinction between "release" and "escape" in producing antiphase solutions. The distinction identifies which of the cells controls the timing of the rapid switches between the active and inactive states. "Release" refers to the case in which the active cell controls the switch, ending its own active state, and thereby releasing the other cell from inhibition at the same time. "Escape" refers to the situation in which the dynamics of the inactive cell allows it to fire in spite of being inhibited; when it becomes active, it shuts off the other cell.

When there are slow synapses, this distinction can no longer be made in general, even when there are antiphase solutions. The inhibition remains after the active cell stops firing, so the "release" mechanism is not relevant. The inhibition decays with a rate having the same order of magnitude as some intrinsic slow processes; when enough inhibition is gone, the intrinsic dynamics, with the remaining amount of inhibition, is oscillatory, and the silent cell can escape. The position at which this happens (if it happens at all) depends on the interaction of all the slow processes.

The analysis of this network illustrates the general theme that when there are several interacting slow processes, either within individual cells [14] or in cells and synapses [28], the network behavior is not apt to be controlled by dynamics of a single cell, but by combinations of processes within and between cells [9, 31]. Furthermore, this "locus of control", or which subset of processes is most important, can change with changes in parameters.

\section{C. Larger networks.}

In another paper, we shall discuss the dynamics of networks of many neurons with inhibitory coupling. Here we just point out some of the consequences of our work on two cells for larger networks of homogeneous cells. It is known from simulations [8, 12] that such inhibitory networks, or networks with both excitatory and inhibitory coupling (see [23, 24]), can display partially synchronized solutions in which "clusters" of cells synchronize, and the clusters remain out of phase with one another. The techniques developed in this paper can be very useful in determining under what conditions the cells within a cluster synchronize and how instabilities within a cluster may arise as parameters are varied.

We note that the clustered solution can appear robustly even when the fully synchronized solution is stable, but has a small domain of stability. Then cells which are not initially sufficiently close get separated, but the cells that are close enough to fire before the onset of inhibition get brought closer together. Within a cluster, the cells both interact with one another and receive input from the rest of population; both effects have been shown to be synchronizing for slowly decaying inhibition. Here we need to assume that the synapses are slowly decaying and indirect; stable clusters cannot form with slowly decaying direct synapses for the same reason why, in this paper, the synchronous solution must be unstable with direct synapses. Stable clusters can form in networks with only fast synapses (because of post inhibitory rebound); however, the synchronous solution cannot be stable in such networks. 
The precise nature of the clustered solution depends on many factors. These include the strength and threshold of the inhibitory coupling, the ratio of the times a single cell spends in the silent and active phase, and the architecture of the network. Some of these issues are discussed in $[6,7,8,23,24]$. Analysis similar to that given for Case 1 in this paper shows how compression or expansion of the cells within a cluster during the silent phase depends on the rate of decay of the synapses and the rate at which individual cells recover in their silent phase. Larger networks may also display a wide assortment of exotic solutions similar to those discussed in Section 4. For example, some of the cells may break up into clusters which take turns firing, while other cells always remain suppressed. There may also be solutions in which the membership of each cluster changes in time. A detailed description of how the emergent behavior of the network depends on all of the parameters is beyond the scope of this paper.

\section{Appendix.}

For the numerics described in Remark 5, Remark 10, and Section 4, we used a system of equations of the form given by (2.4). This system is similar to the model of Morris and Lecar [17]. We let

$$
\begin{aligned}
& f(v, w)=.5(v+.5)-3 w\left(v+\alpha_{1}\right)-\alpha_{2} m_{\infty}(v)\left(v-\alpha_{5}\right)+.2 \\
& g(v, w)=\left(\gamma w_{\infty}(v)-w\right) / \tau(v)
\end{aligned}
$$

Here,

$$
\begin{aligned}
m_{\infty}(v) & =.5(1+\tanh ((v+.01) / .15)) \\
w_{\infty}(v) & =.5\left(1+\tanh \left(\left(v-\alpha_{3}\right) / .002\right)\right) \\
\tau(v) & =1 / \cosh \left(\left(v-\alpha_{4}\right) / .29\right)
\end{aligned}
$$

For direct synapses, we used an equation of the form (2.5), except we replaced the Heaviside function by $s_{\infty}(v)=\left\{1+\exp \left(-\left(v_{j}-\theta_{\text {syn }}\right) / .001\right)\right\}^{-1}$. For indirect synapses, we used equations of the form given in (2.6), except we replaced $H\left(v_{j}-\theta_{v}\right)$ by $x_{\infty}(v)=\{1+$ $\left.\exp \left(-\left(v_{j}-\theta_{v}\right) / .001\right)\right\}^{-1}$ and we replaced $H\left(x_{i}-\theta_{\text {syn }}\right)$ by $s_{\infty}(v)=\left\{1+\exp \left(-\left(x_{i}-\right.\right.\right.$ $\left.\left.\left.\theta_{\text {syn }}\right) / .001\right)\right\}^{-1}$.

Acknowledgment:. We wish to thank Carson Chow for many helpful discussions about integrate and fire neurons, Eve Marder for discussions about "locus of control" in neural systems, and J. Rinzel and G. B. Ermentrout for conversations about slow inhibition. 


\section{References}

1. P. Bush and T. Sejnowski, "Inhibition synchronizes sparsely connected cortical neurons within and between columns of realistic network models." J. Comp. Neurosci. 3 (1996) 91-110.

2. A. Destexhe, D. Contreras, T.J. Sejnowski and M. Steriade, "A model of spindle rhythmicity in the isolated thalamic reticular nucleus," J. Neurophysiol. 72 (1994) 803-818.

3. U. Ernst, K. Pawelzik, and T. Giesel. Synchronization induced by temporal delays in pulse-coupled oscillators. Phys. Rev. Lett. 74 (1995) 1570-1573.

4. W. Friesen, "Reciprocal inhibition", a mechanism underlying oscillatory animal movements", Neurosci. Behavior 18 (1994) 547-553.

5. W. Gerstner, J.L. van Hemmen, and J. Cowen. What matters in neuronal locking? Neural Comp. 8 (1996) 1653-1676.

6. D. Golomb and J. Rinzel, "Dynamics of globally coupled inhibitory neurons with heterogeneity", Phys. Rev. E 48 (1993) 4810-4814.

7. D. Golomb and J. Rinzel, "Clustering in globally coupled inhibitory neurons", Physica D 72 (1994) 259-282.

8. D. Golomb, X-J Wang and J. Rinzel, "Synchronization properties of spindle oscillations in a thalamic reticular nucleus model", J. Neurophys. 72 (1994).

9. S.L. Hooper and M. Moulins, "Switching of a neuron from one network to another by sensory-induced changes in membrane properties", Science 244 (1989) 1587-1589.

10. J.G.R. Jeffreys, R.D. Traub and M.A. Whittington. "Neuronal networks for induced '40 Hz' rhythms," Trends Neurosci. 19, (1996) 202-207.

11. N. Kopell, "Toward a theory of modeling central pattern generators, in Neural Control of Rhythmic Movements in Vertebrates (A.H. Cohen, S. Rossignol and S. Grillner, eds.) Wiley N.Y., 1987, pp. 369-413.

12. N. Kopell and G. LeMasson, "Rhythmogenesis, amplitude modulation and multiplexing in a cortical architecture,", Proc. Nat. Acad. Sci (USA) 91 (1994) 10586-10590.

13. T. LoFaro, N. Kopell, E. Marder and S. Hooper, "Subharmonic coordination in networks of neurons with slow conductances", Neural Computation, 6 (1994) 69-84.

14. T. LoFaro and N. Kopell, "Time regulation in a network reduced from voltage-gated equation to a one-dimensional map", submitted

15. W. Lytton and T. Sejnowski, "Simulations of cortical pyramidal neurons synchronized by inhibitory neurons", J. Neurophysiol. 66 (1991) 1059-1097. 
16. E.F. Mishchenko and N.Kh. Rozov, Differential Equations with Small Parameters and Relaxation Oscillations, Plenum Press, New York and London (1980)

17. C. Morris and H. Lecar, "Voltage oscillations in the barnicle giant muscle fiber", Biophysical J. 35 (1981) 193-213.

18. D. Perkel and B. Mulloney, "Motor pattern production in reciprocally inhibitory neurons exhibiting postinhibitory rebound", Science 185 (1974) 181-183.

19. P. Rowat and A. Selverston, "Oscillatory mechanisms in pairs of neurons connected with fast inhibitory synapses J. Computational Neuroscience, 4 (1997) 103-127.

20. F. Skinner, N. Kopell and E. Marder, "Mechanisms for oscillation and frequency control in networks of mutually inhibitory relaxation oscillators", J. Computational Neuroscience, 1 (1994) 69-87.

21. D. Somers and N. Kopell. Rapid synchronization through fast threshold modulation. Biol. Cyber. 68, 393-407 (1993).

22. M. Steriade, E.G. Jones and R.R. Llinás. Thalamic oscillations and signaling. Wiley, New York, 1990.

23. D. Terman and E. Lee, "Partial synchronization in a network of neural oscillators", to appear in SIAM J. Appl. Math.

24. D. Terman and D.L. Wang, "Global competition and local cooperation in a network of neural oscillators, Physica D 81 (1995) 148-176.

25. C. van Vreeswijk, "Partial synchronization in populations of pulse-coupled oscillators", Phys. Rev. E 54 (1996) 5522-5537.

26. C. van Vreeswijk, L. Abbott and G.B. Ermentrout, "When inhibition, not excitation synchronizes neural firing", J. Comp. Neuroscience (1994) 313-321.

27. X.J. Wang and G. Buzsaki, "Gamma oscillation by synaptic inhibition in an interneuronal network", J. Neurosci. 1996.

28. X.J. Wang and J. Rinzel, "Alternating and synchronous rhythms in reciprocally inhibitory model neurons", Neural Comp. 4 (1992) 84-97.

29. X.J. Wang and J. Rinzel, "Spindle rhythmicity in the reticularis thalami nucleus: synchronization among mutually inhibitory neurons", Neuroscience 53 (1993) 899-904.

30. X.-J. Wang, D. Golomb, and J. Rinzel. Emergent spindle oscillations and intermittent burst firing in a thalamic model: specific neuronal mechanisms. Proc. Natl. Acad. Sci. (USA) 92 (1995) 5577-5581. 
31. J.M. Weimann, P. Meyrand and E. Marder, "Neurons that form multiple pattern generators: Identification and multiple activity patterns of gastric/pyloric neurons in the crab stomatogastric system", J. Neurophysiol. 65 (1991) 111-122.

32. M.A. Whittington, R.D. Traub, and J.G.R. Jefferys. Synchronized oscillations in interneuron networks driven by metabotropic glutamate receptor activation. Nature $\mathbf{3 7 3}$ (1995) 612-615.

33. J. White, C.C. Chow, J. Ritt, C. Soto and N. Kopell, "Synchronization and oscillatory dynamics in heterogeneous, mutually inhibited neurons", to appear in J. Computational Neuroscience.

34. C.C. Chow and J. Ritt, "Phaselocking in weakly heterogeneous neuronal networks", preprint. 


\section{Figure Captions}

Figure 1: Nullclines of (2.1). The singular periodic orbit is shown with a bold curve.

Figure 2. The slow phase space. Each trajectory corresponds to a solution of (2.9). (A) If a single cell is oscillatory, then every trajectory must leave the silent phase along the curve of knees $w=w_{L}(s)$ and then jump up to the active phase (denoted by the ${ }^{*}$ ). (B) If a single cell is excitable, then some trajectories must approach the stable fixed point along the line $s=0$. Other trajectories, such as that denoted with a dashed curve, may still leave the silent phase.

Figure 3. The slow phase space when $K$ is small. (A) Here $K=0$, and a single cell is excitable. The critical points of (2.9) consist of the two curves $w_{c}^{+}$and $w_{c}^{-}$. In this example, there are levels of inhibition (values of $s$ between the curves) for which the cells are oscillatory. (B) If $K$ is sufficiently small, then the synchronous solution will exist although the single cell is excitable. The synchronous solution will cross $w_{c}^{+}$and leave the silent phase through the curve of knees $w_{L}(s)$.

Figure 4. The synchronous solution is unstable for direct synapses. Here the two cells begin close to each other in the silent phase. Cell 1 reaches the curve of knees first and jumps up. This immediately (on the slow time scale) resets the inhibition of cell 2 to $s=1$.

Figure 5. Geometric illustration of the proof of Proposition 1. Each cell's inhibition begins to decrease when $\tau=t_{i}$ and the cell leaves the silent phase when $\tau=T_{i}$. The dashed curve is a time translate of the curve of knees.

Figure 6. Geometric illustration of the jump up for Case 1. Here cell 1 jumps up before cell $2\left(T_{1}<T_{2}\right)$ and cell 1 is 'ahead' of cell 2 on the right branch after both cells have jumped up $\left(w_{2}\left(T_{2}\right)<w_{1}\left(T_{2}\right)\right)$.

Figure 7. Common inhibitory input can help synchronize unconnected cells if the inhibition decays at appropriate rates. The two cells have different (but similar) frequencies, start synchronously and were allowed to run with A) common decaying inhibition and B) common constant inhibition. Note that the decaying inhibition keeps the two cells more synchronous.

Figure 8. Geometric illustration of the jump up for Case $2 \mathrm{~A}$. Here cell 1 jumps up before cell $2\left(T_{1}<T_{2}\right)$ and cell 1 is 'behind' cell 2 on the right branch after both cells have jumped up $\left(w_{1}\left(T_{2}\right)<w_{2}\left(T_{2}\right)\right)$.

Figure 9. Geometric illustration of the jump up for Case 2B. Here cell 1 jumps up after cell $2\left(T_{1}>T_{2}\right)$. 
Figure 10. Geometric illustrations for the case $t_{1}<t_{2}$. Then cell 1 's inhibition begins to decrease before that of cell 2. The dashed curves are time translates of the curve of knees. (A) If $t_{2}-t_{1}<\delta$, then cell 2 lies to the 'right' of cell 1 . (B) If $t_{2}-t_{1}>\delta$, then cell 1 lies to the 'right' of cell 2.

Figure 11. (A) Suppressed and (B) antiphase solutions of the network. Time is measured in $\mathrm{ms}$.

Figure 12. The projection of the antiphase solution onto the slow phase space. One half of a complete cycle is shown. Cell 1 always lies in the silent phase and is illustrated with a solid curve. Cell 2 begins in the silent phase and jumps up at time $\tau_{1}$. Its trajectory during the silent phase is illustrated with a dashed curve and during the active phase it is illustrated with a dotted curve.

Figure 13. An exotic solution of (2.1). One cell fires three time while the other is silent, and then the roles of the cells are reversed.

Figure 14. Another exotic solution of (2.1). One cell fires twice for every one time the other cell fires. 